\title{
Recent Higgs Results from the ATLAS experiment
}

\author{
Kurt Brendlinger \\ On behalf of the ATLAS Collaboration
}




\section{Outline}

The Discovery of the Higgs Boson

- Production mechanisms, cross sections and branching ratios of the Higgs at the LHC

- The ATLAS Detector

Higgs Boson "Discovery Channels"

- $\mathrm{H} \rightarrow \mathrm{Yr}$ Channel

- $\mathrm{H} \rightarrow \mathrm{ZZ}$ Channel

- H $\rightarrow$ WW Channel

Other Higgs channels

- $\mathrm{H} \rightarrow \mathrm{TT}$

- $\mathrm{ZH} \rightarrow \|+$ invisible

- $\mathrm{H} \rightarrow$ bb Channels

\section{Higgs Boson Properties}

- Signal Strength and Couplings

- Spin

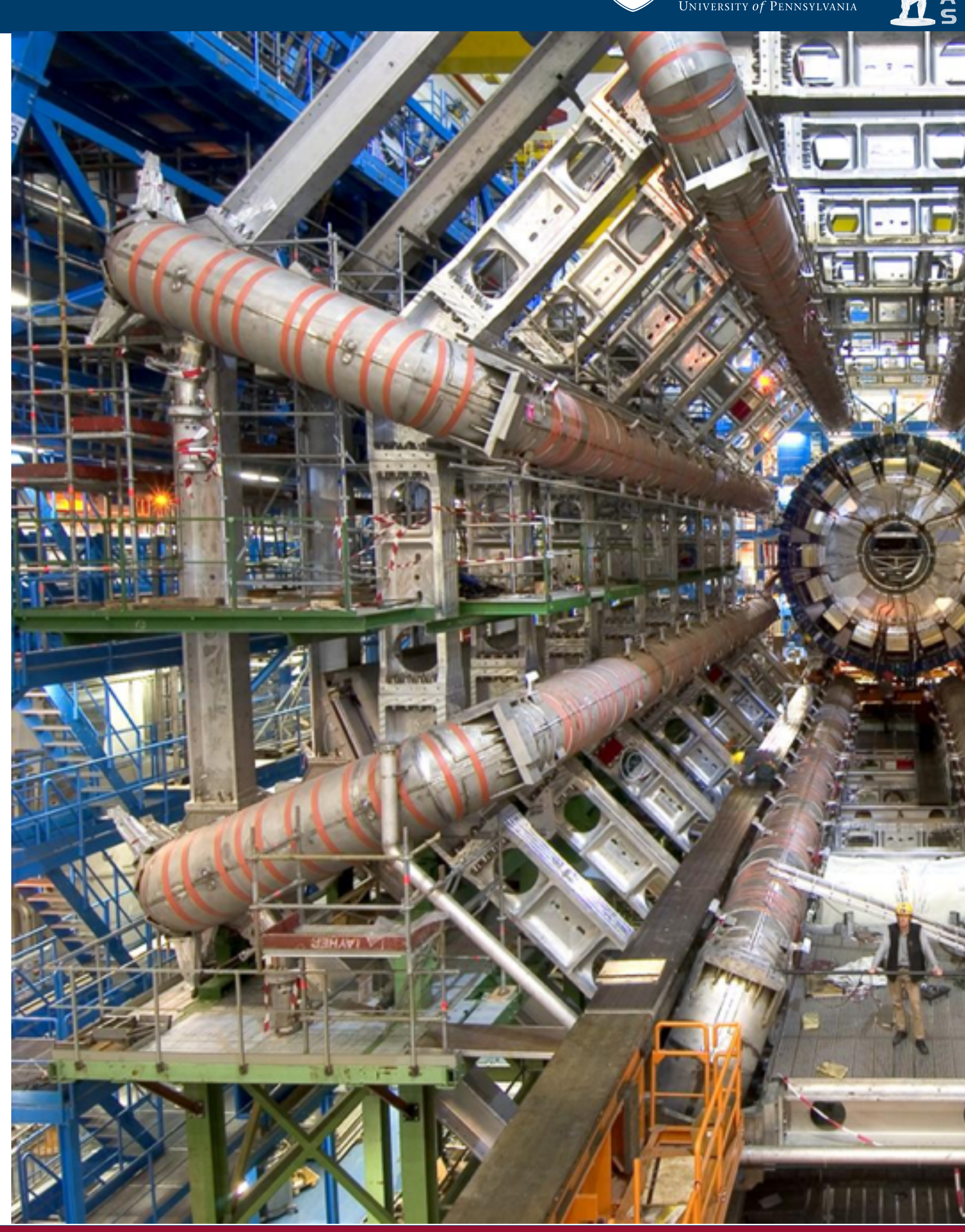




\section{Higgs Boson Production Modes}

- Main production: gluon-gluon fusion

- Proceeds mainly through the top loop

- Roughly I0x the production $\left(@ M_{\mathrm{H}}=125 \mathrm{GeV}\right)$ at the LHC than at the Tevatron

\section{- Vector Boson Fusion}

- Lower cross section

- Involves direct probe into vector boson coupling constant

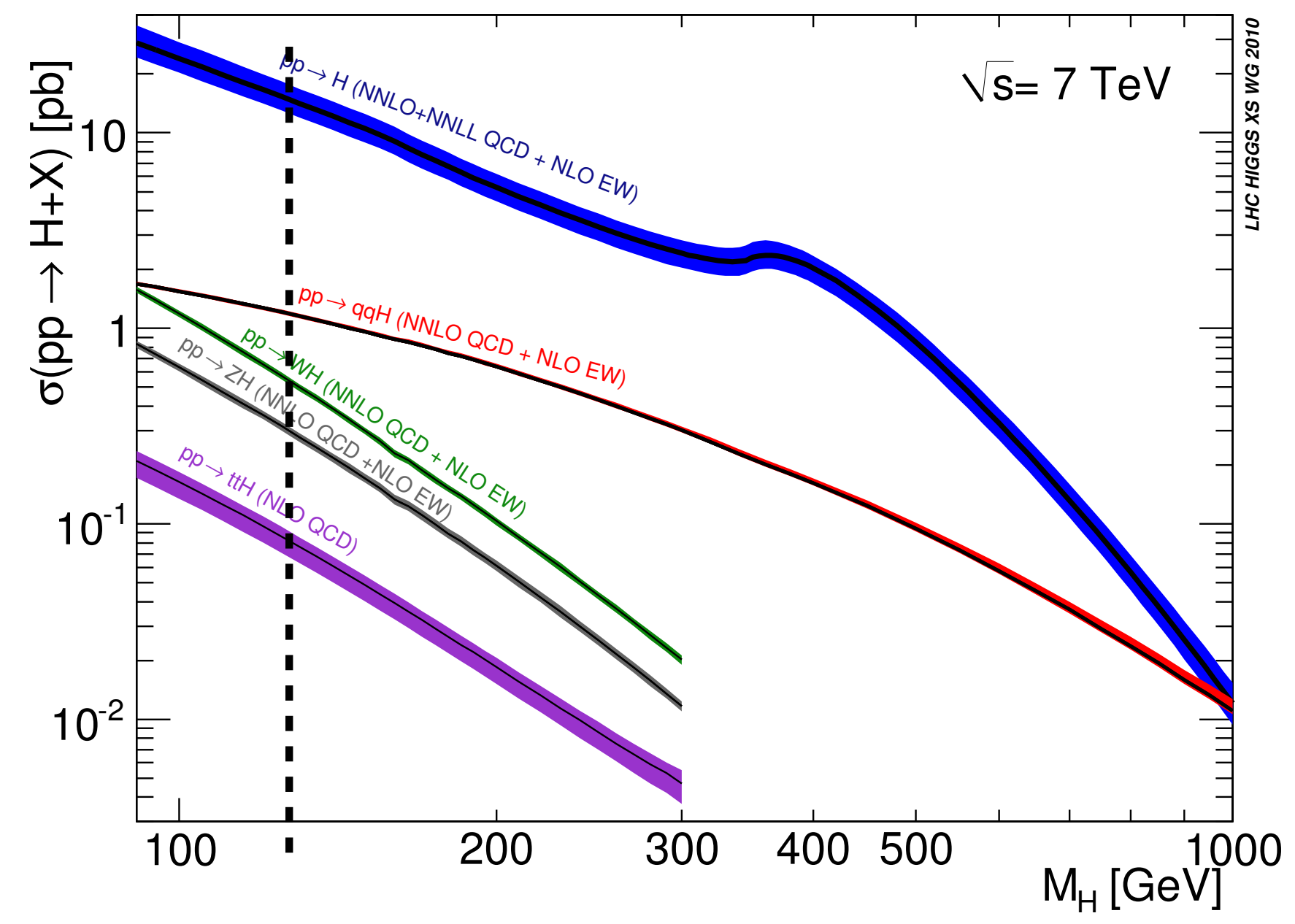

- Signature includes two forward high-Pt jets with a large separation in rapidity space

- Analyses use this signature to target this production mode

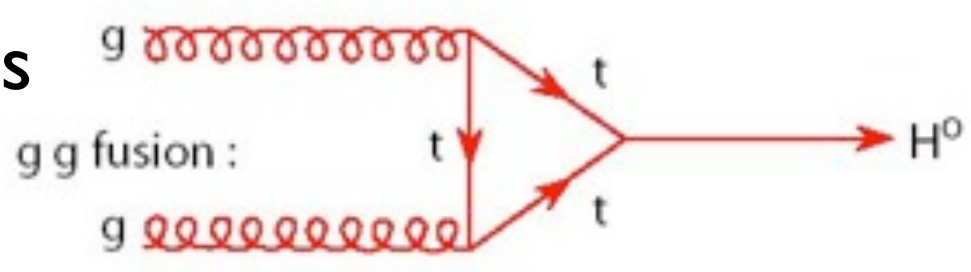

- $\mathrm{V} \rightarrow \mathrm{VH}$ (associated production)

- ttbar fusion

- Direct coupling to top quark
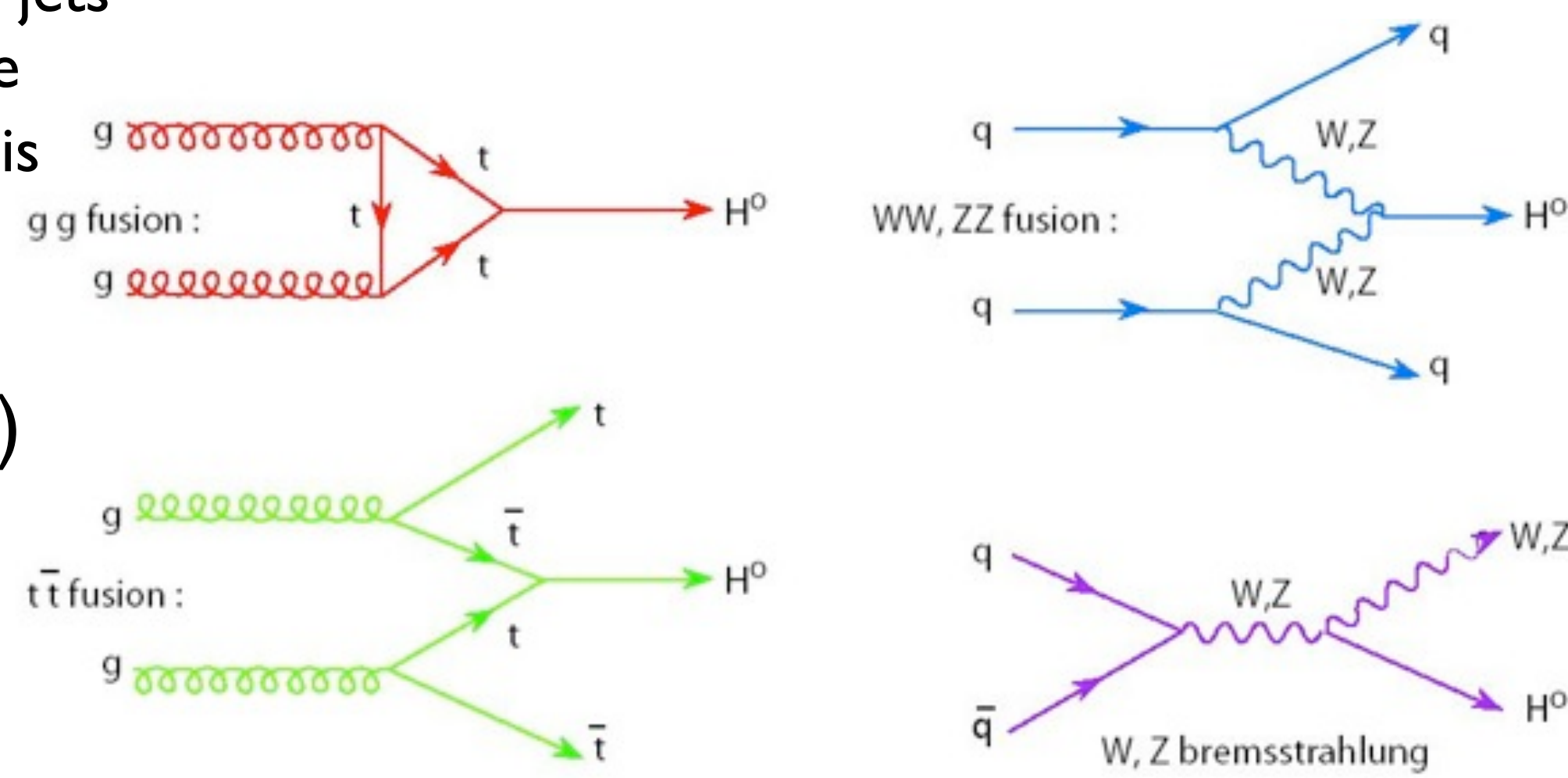

http://sites.uci.edu/energyobserver/2012// |/26/higgs-production-and-decay-channels/

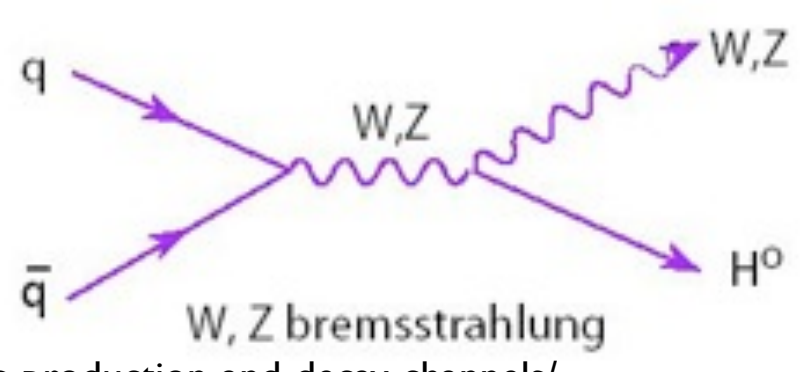




\section{Higgs Final States}

\section{Guiding principles}

- Final states with leptons or photons are easier to distinguish, measure

- Decays to jets are more difficult to separate from QCD background

\section{Discovery Channels}

- $\mathrm{H} \rightarrow \mathrm{YY}$

- $\mathrm{H} \rightarrow \mathrm{ZZ}^{*} \rightarrow 4 \mathrm{I}$

- $\mathrm{H} \rightarrow \mathrm{WW}^{*}(\rightarrow \mid \mathrm{vlv})$

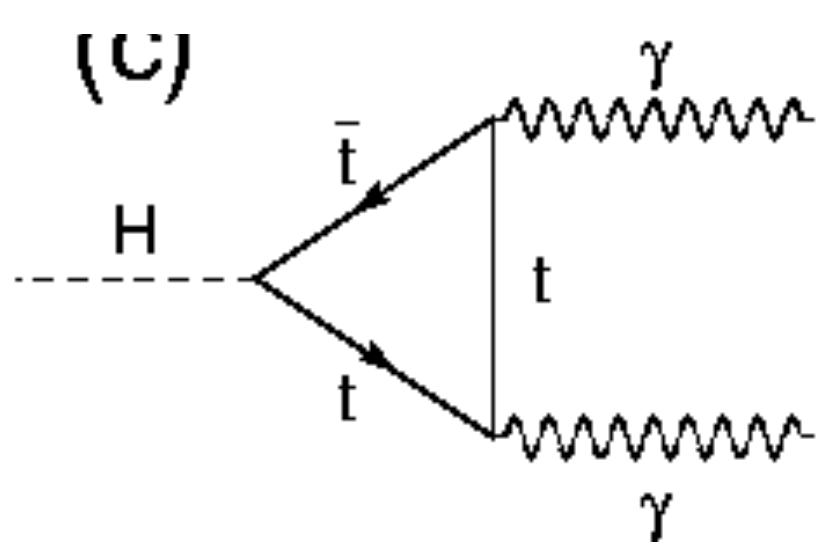

$\overline{0}$
$\stackrel{0}{2}$
$\frac{\pi}{0}$
$\times$
0

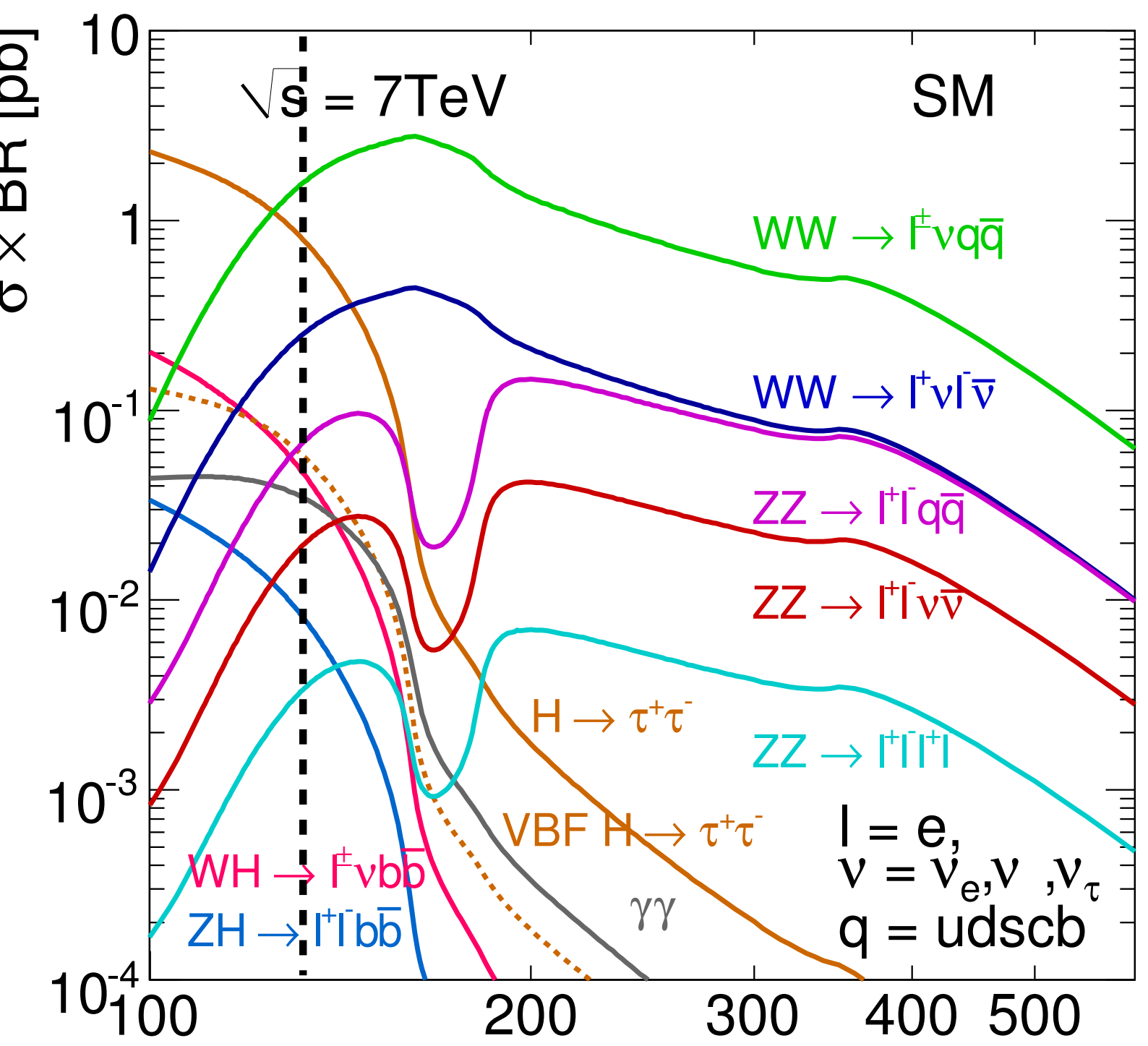

\section{Additional Channels}

- $\mathrm{H} \rightarrow \mathrm{T \tau}$

- $\mathrm{H} \rightarrow \mu \mu$

- $\mathrm{ZH} \rightarrow \|+$ Invisible

- $\mathrm{H} \rightarrow$ bb

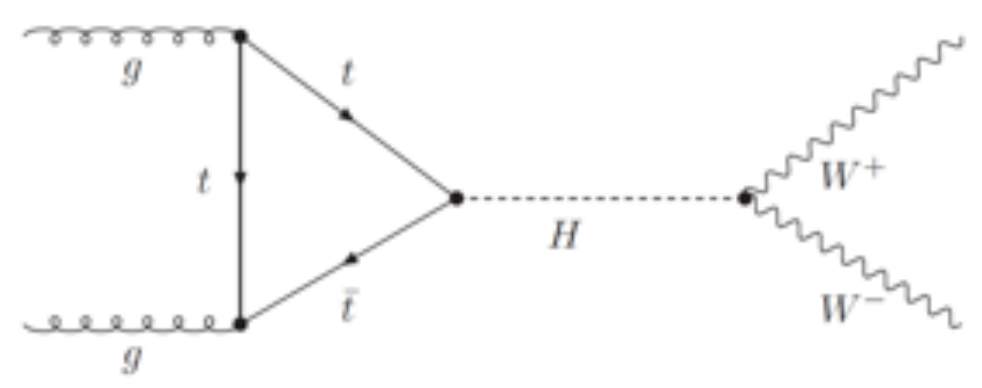
$\mathrm{M}_{\mathrm{H}}[\mathrm{GeV}]$ 


\section{The LHC}

- The LHC:

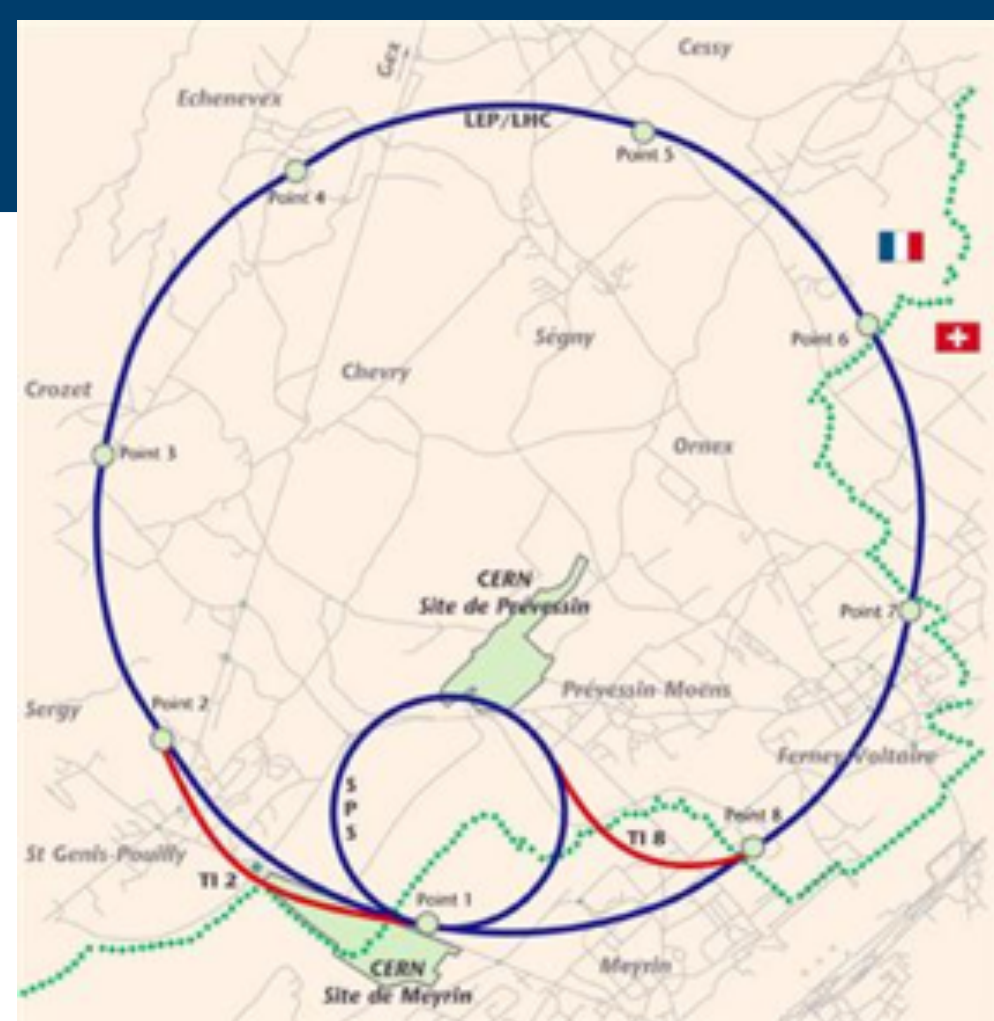

\section{Phenomenal Machine Performance}

- Proton-proton collider

- CM Energies of $7 \mathrm{TeV}(20 \mathrm{II})$ and $8 \mathrm{TeV}(20 \mathrm{I} 2)$

- Delivered $\sim 5 \mathrm{fb}^{-1}$ in $20 \mathrm{II}$

- Delivered $23 \mathrm{fb}^{-1}$ in 2012

- Peak instantaneous luminosity: $\sim 8 \times 10^{33} \mathrm{~cm}^{-2} \mathrm{~s}^{-1}$

- Challenge in 2012: High pileup conditions

- Average interactions per bunch crossing increased

- Must reconstruct and resolve 20 vertices within the space of $\sim 100$ s of $\mathrm{mm}$

- Requires careful treatment of missing transverse momentum calculation
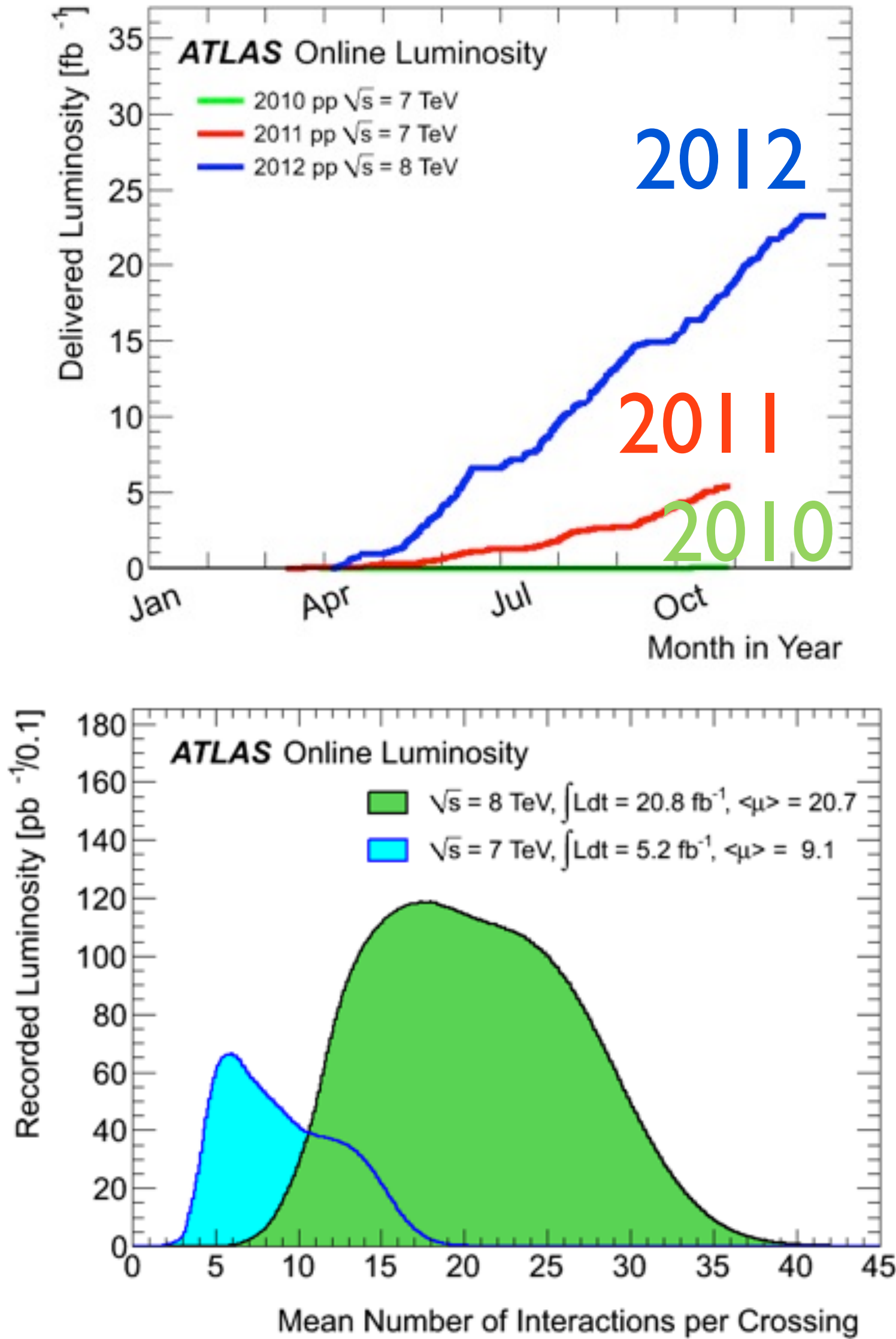


\section{The ATLAS Detector}

\section{The ATLAS detector was built with the discovery of the Higgs in mind}

- Identification of electrons and photons against $Q C D$ jets

- Precise measurement of particle energy and position

- Excellent calorimetry hermeticity and depth for inferring momentum imbalance (missing transverse momentum)

- Precise muon spectrometry and triggering

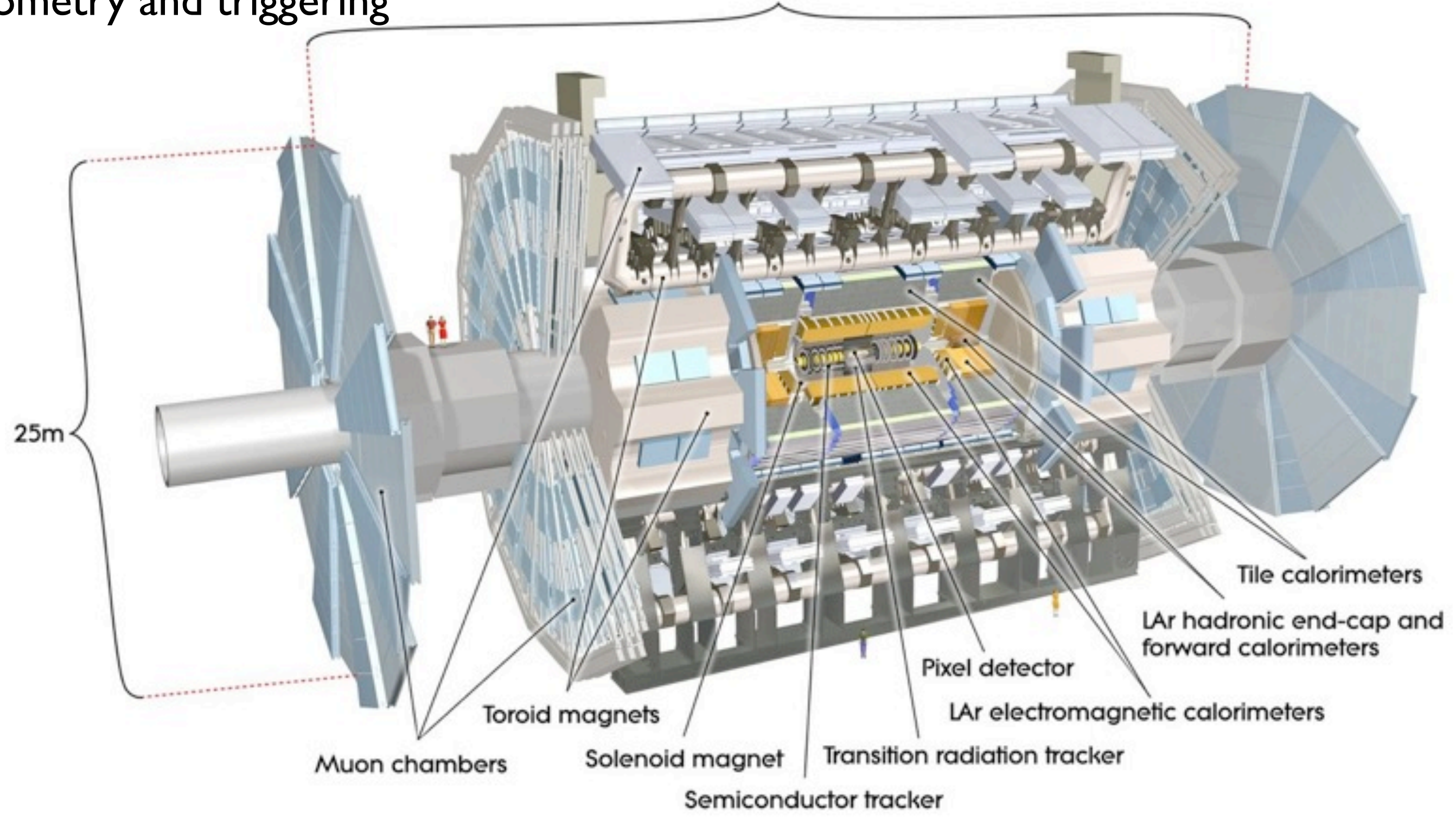




\section{The ATLAS Detector}

The ATLAS detector was built with the discovery of the Higgs in mind

- The Inner Detector

- Provides tracking of charged particles

- 2T magnetic field

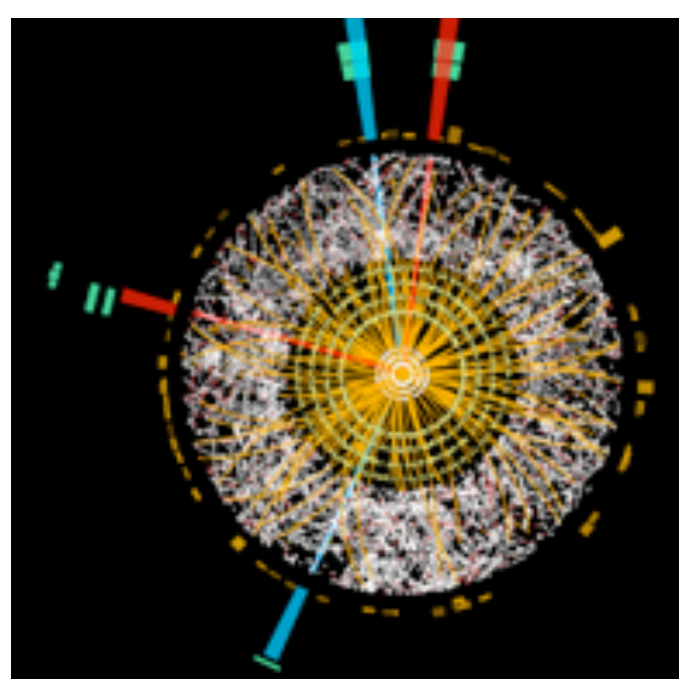

- Silicon pixel detector: 80M channels, $10 x \mid 15 \mu \mathrm{m}$ spatial resolution

- SCT microstrips: $6 \mathrm{M}$ channels, $17 \mu \mathrm{m}$ resolution

- TRT: Particle identification of electrons using transition radiation

- The Calorimeter Systems

- Energy measurement and detailed shower shapes for particle identification

- Fine segmentation of the first calorimeter layer to determine the kinematics of photon path

- Full volume coverage to measure missing transverse momentum in an event

- The Muon Spectrometer

- Magnetic field generated by toroidal superconducting air-core magnets

- Separate systems for precise tracking and triggering

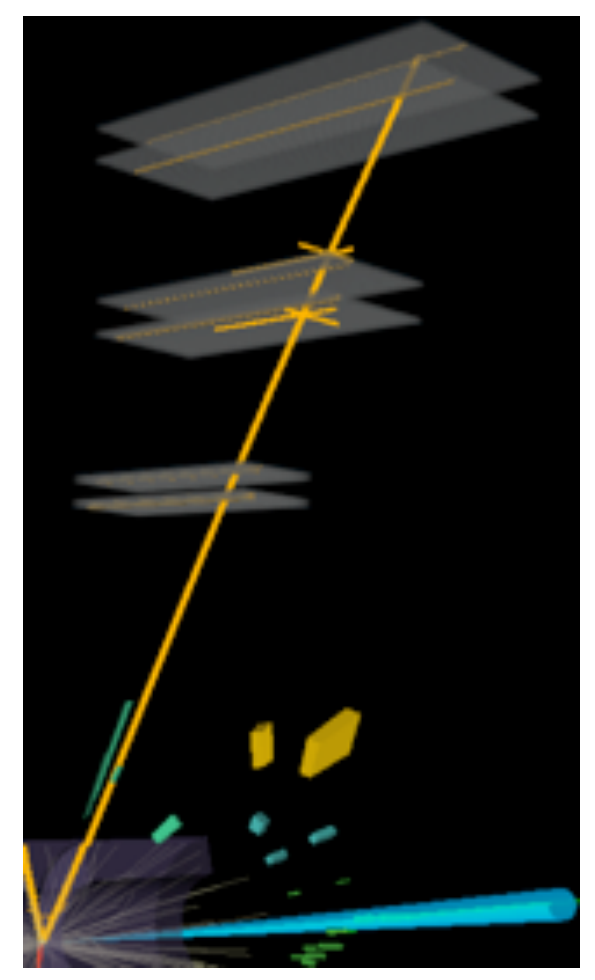


- Discovery driven by $\mathrm{H} \rightarrow \mathrm{YY}, \mathrm{H} \rightarrow \mathrm{ZZ}$, $\mathrm{H} \rightarrow$ WW channels

- How did we get there?

- Is it the Higgs Boson?

\section{What can we measure?}

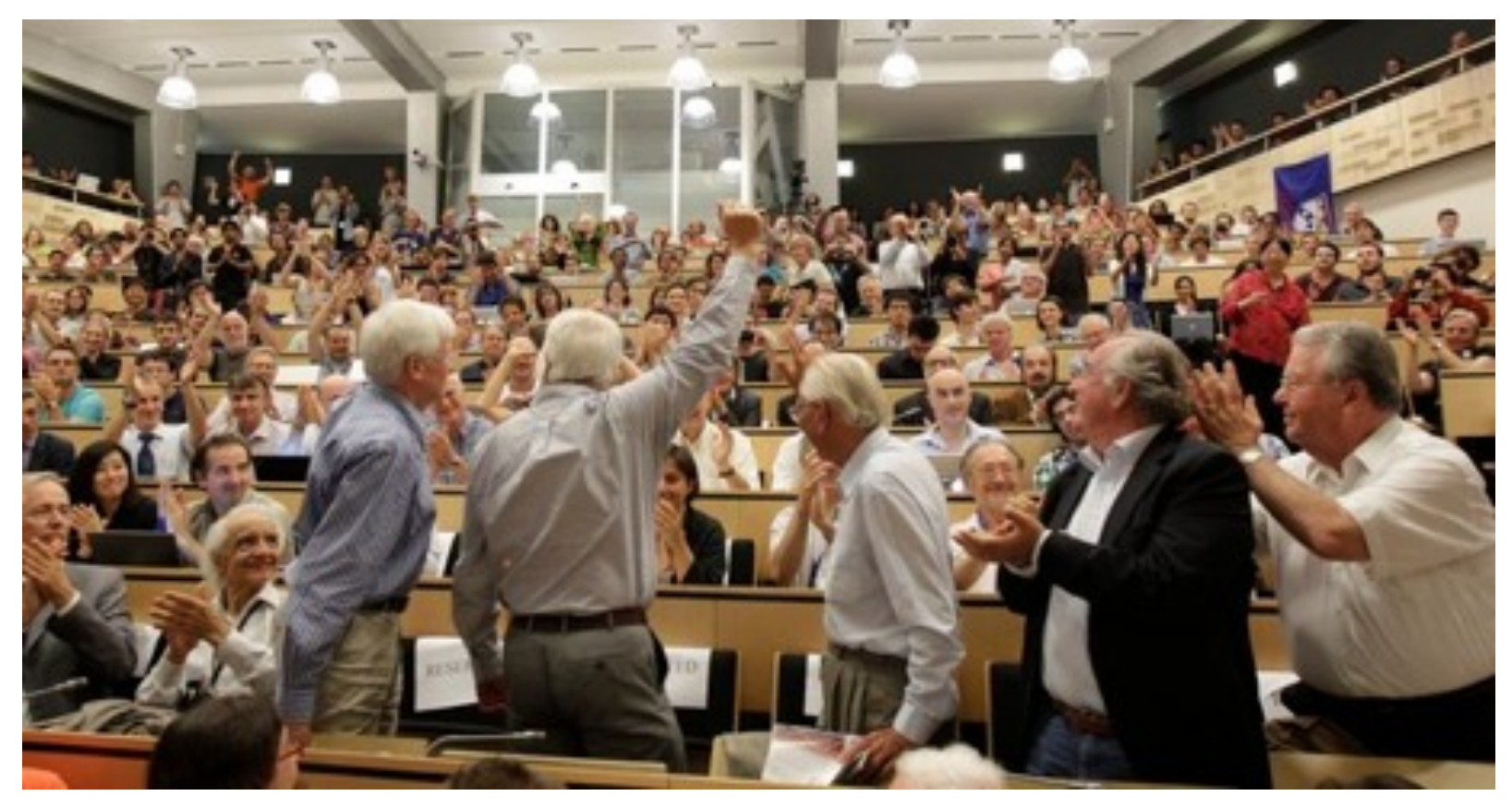

- Test signal strength compatibility with standard model: $\mu=\frac{\sigma \times B R}{\sigma_{S M} \times B R_{S M}}$

- Measure Higgs couplings - test their compatibility with the SM

- Test particle spin/parity hypotheses (expect that the Higgs is a scalar CPeven particle)

- Probe decays to fermionic final states

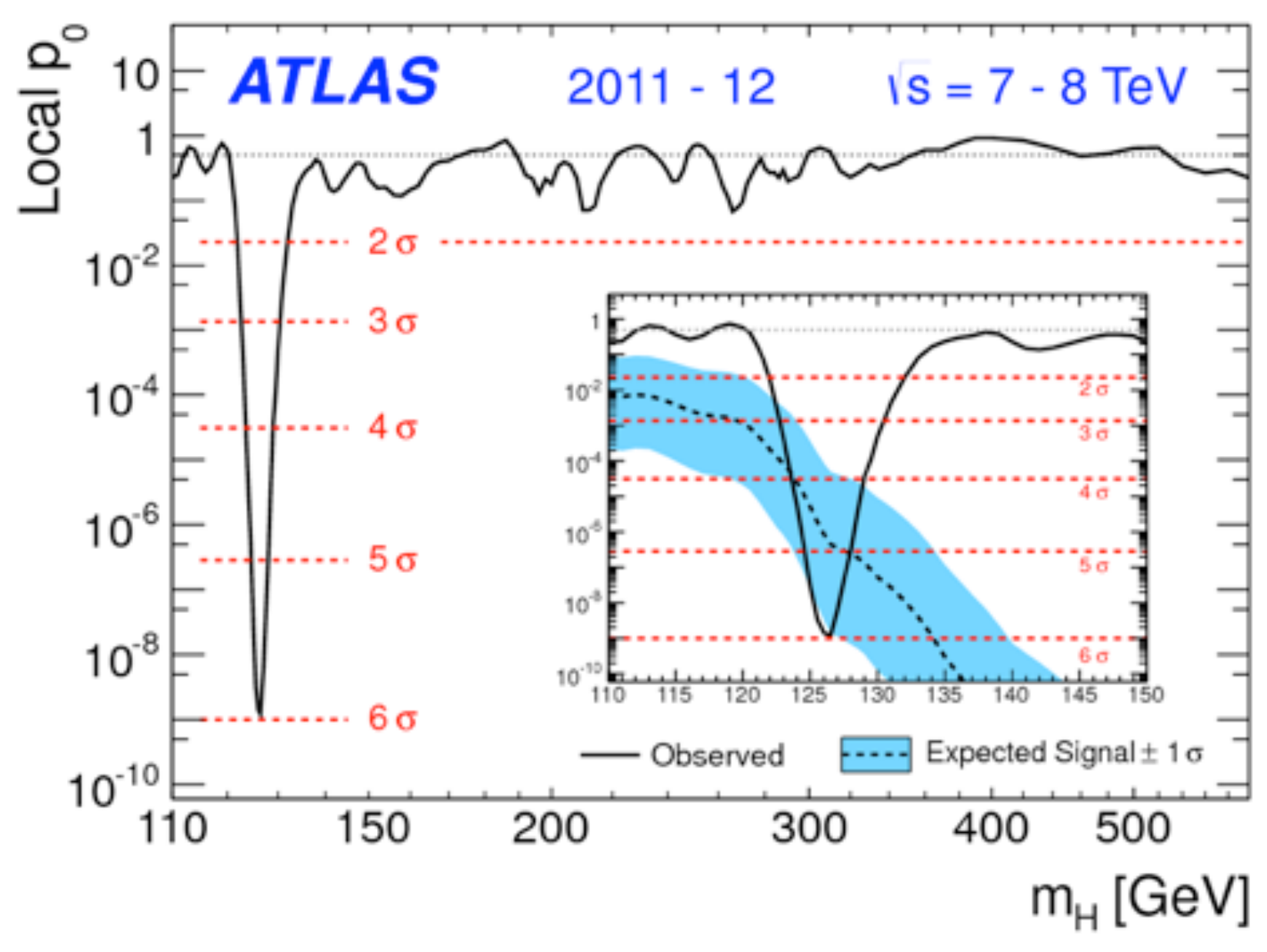


- Dominant production mode is gluon fusion: $87 \%$

- Background

- Irreducible VY production, and reducible $\gamma$-jet production

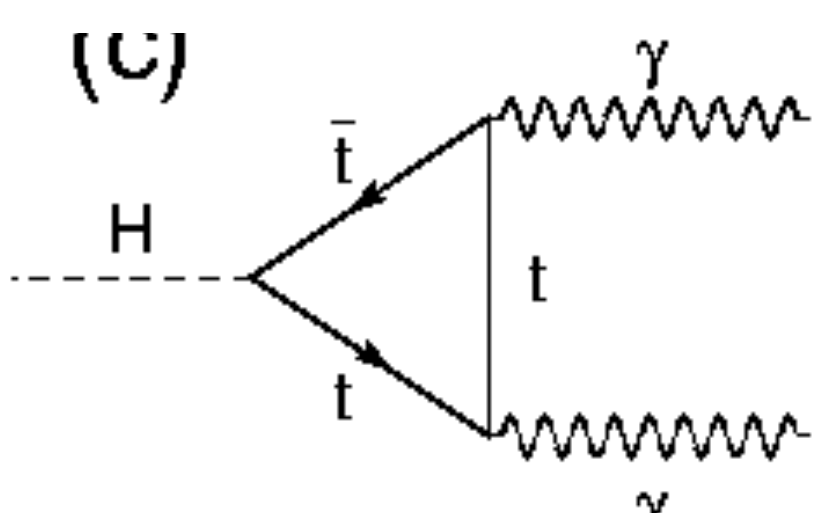

- Smooth shape; characterized by analytic functions

- Categorize analysis to separate production modes, backgrounds

- Separate VBF, WH/ZH, ggF categories

- Further separation into categories of converted and unconverted photons

- Increases sensitivity

- Measurements

- Mass: I 26.8 \pm 0.2 (stat) \pm 0.7 (syst) GeV

- Total $\mu=1.65 \pm 0.24$ (stat)+0.25-0. 18(syst)

- Separate signal strength measurements for $\mu_{g g F+t t H}, \mu_{\vee B F}$, $\mu_{\mathrm{VH}}$

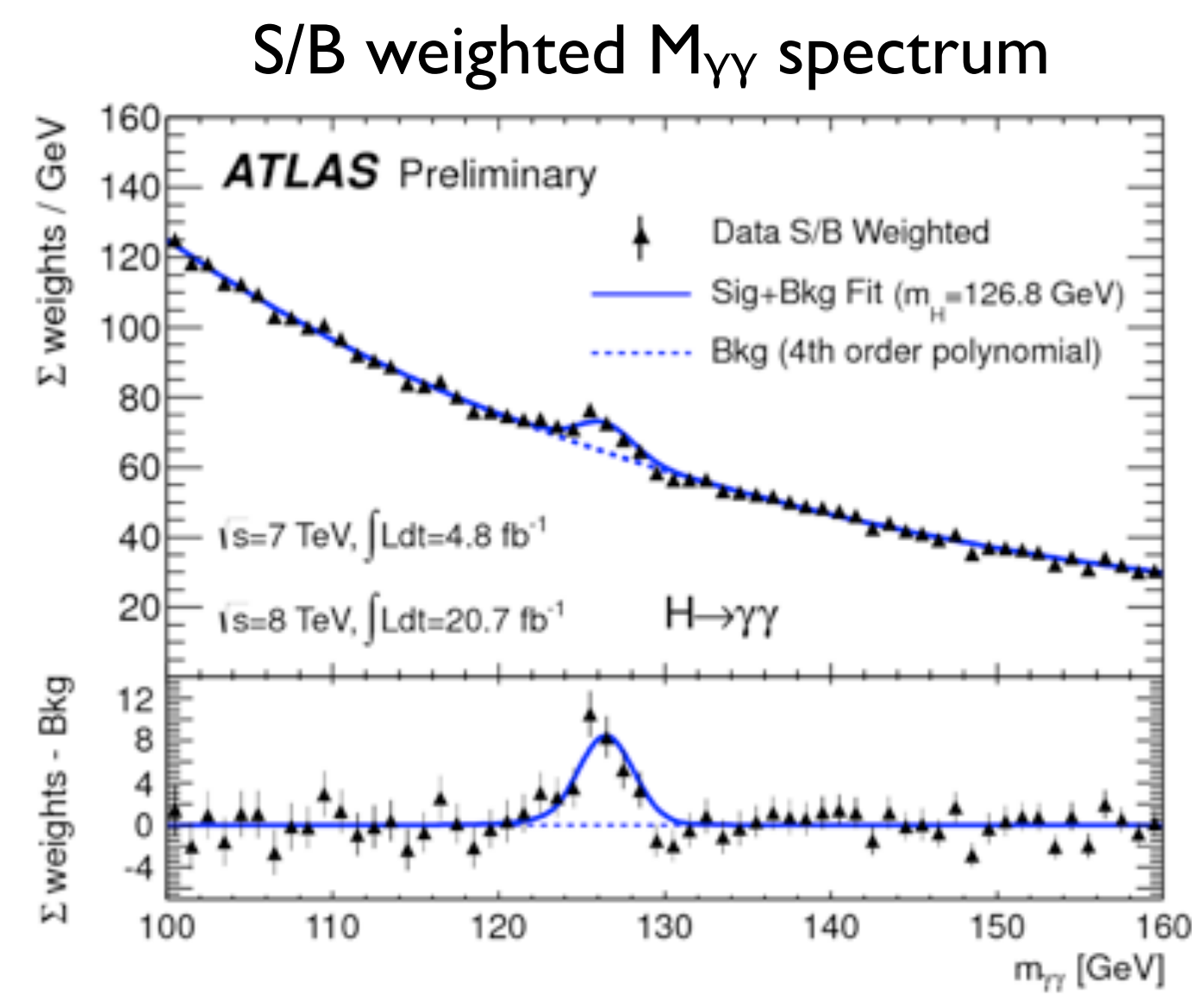

- local significance of $7.4 \sigma$ at $\mathrm{mH}_{\mathrm{H}}=126.5 \mathrm{GeV}$

ATLAS-CONF-2013-029 


\section{$\mathrm{H} \rightarrow \mathrm{ZZ} \rightarrow 4$ e Candidate}

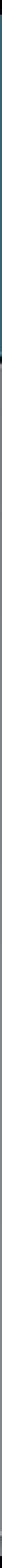




\section{$\mathrm{H} \rightarrow \mathrm{ZZ} *$ Channel}

- Decay to an on-shell Z and an off-shell Z

- Cleanest channel - but also very low $x$ sec $\bullet B R$

- Statistics-limited analysis

- Largest irreducible bkg: SM ZZ production

- Peak at $90 \mathrm{GeV}$ is single-resonant $Z$ peak

- Largest reducible bkg: ttbar, Z+jets

- Separate treatment of $4 \mu, 2 \mu 2 \mathrm{e}, 2 \mathrm{e} 2 \mu$ and $4 \mathrm{e}$ channels

- Separation into ggF,VBF and VH-like categories

- Measurements

- Mass Measurement is driven by $4 \mu, 2 e 2 \mu$ channels

- $\mathrm{m}_{\mathrm{H}}=124.3+0.6-0.5$ (stat)+0.5-0.3 (syst) GeV

- Signal strength: $\mu=1.7+0.5-0.4$

- Significance: $6.6 \sigma$
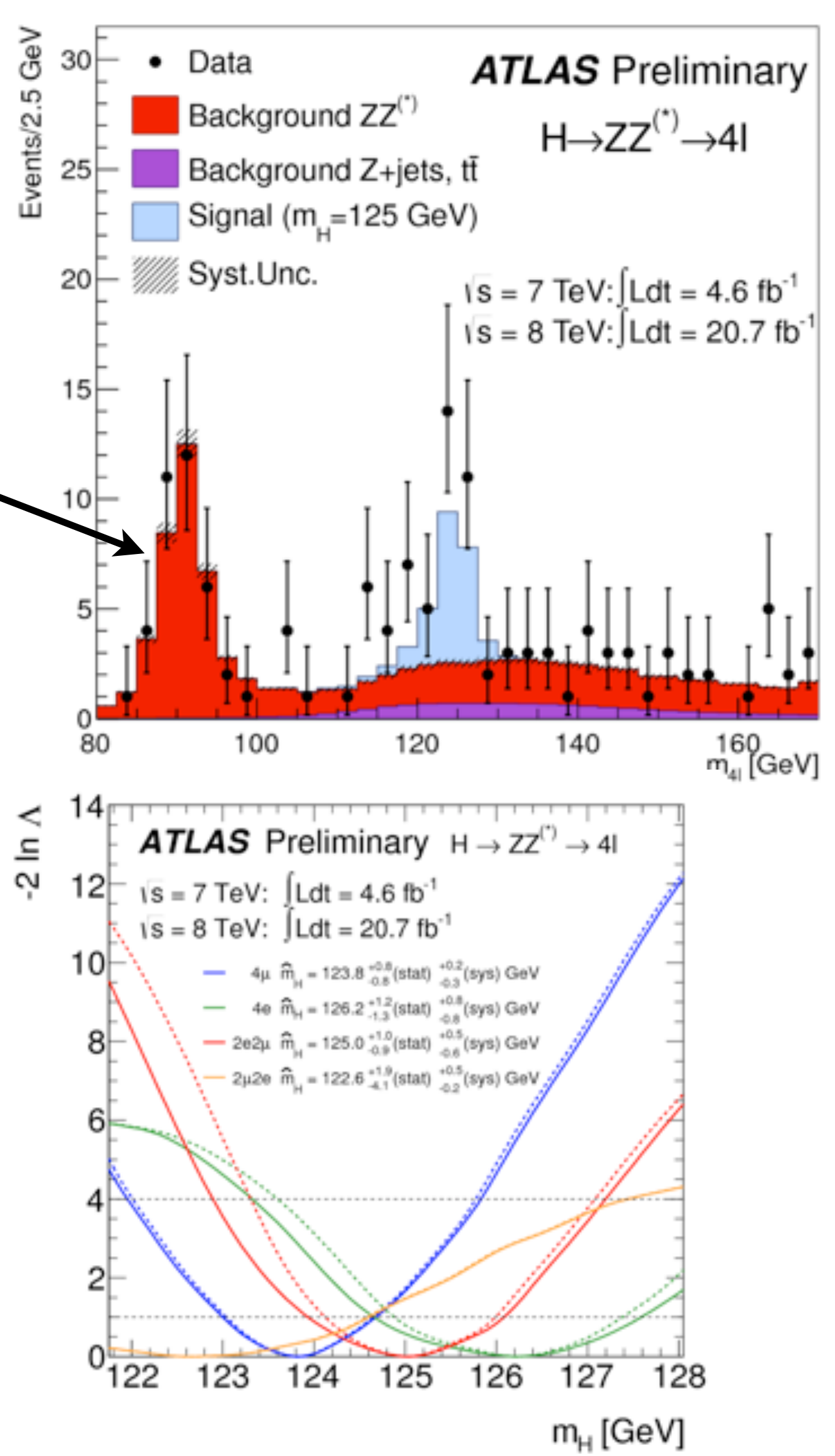
17 Nov 2012 07:42:05 CET

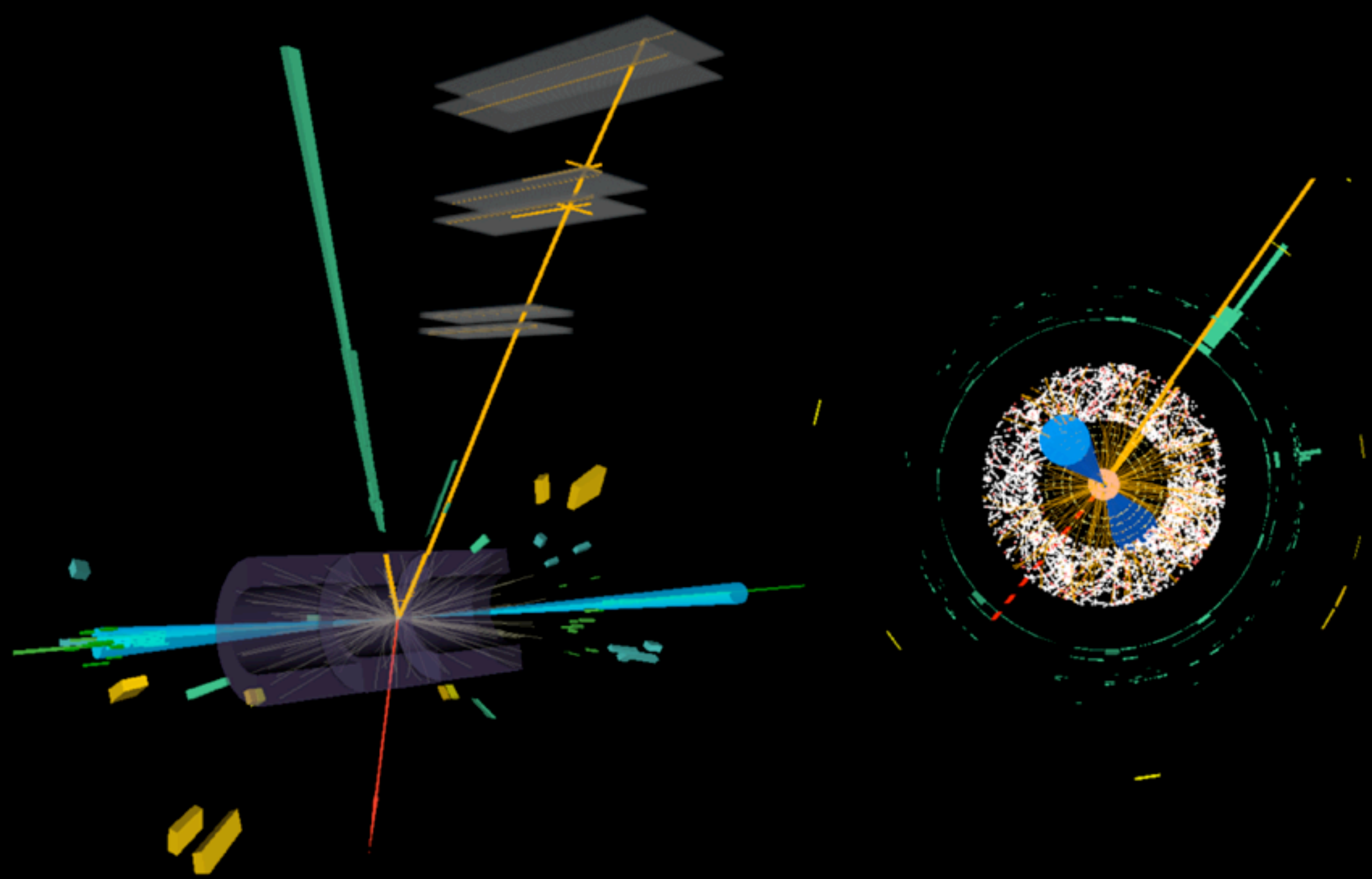


- Presence of two neutrinos prevents full reconstruction of event kinematics

- Instead look at the transverse mass variable, MT

- Largest irreducible bkg: SMWW production

- Other bkg contributions from ttbar, $W+j e t s$

- Split analysis into Njet = I, Njet $=2$

- Goal is to split backgrounds for higher significance

- VBF channel: Njet $\geq 2$ Measurements

- Signal strength: $\mu=1.0 \mathrm{I} \pm 0.3 \mathrm{l}$.

- Significance: $3.8 \sigma$ excess overall

- VBF channel: $2.5 \sigma$ excess

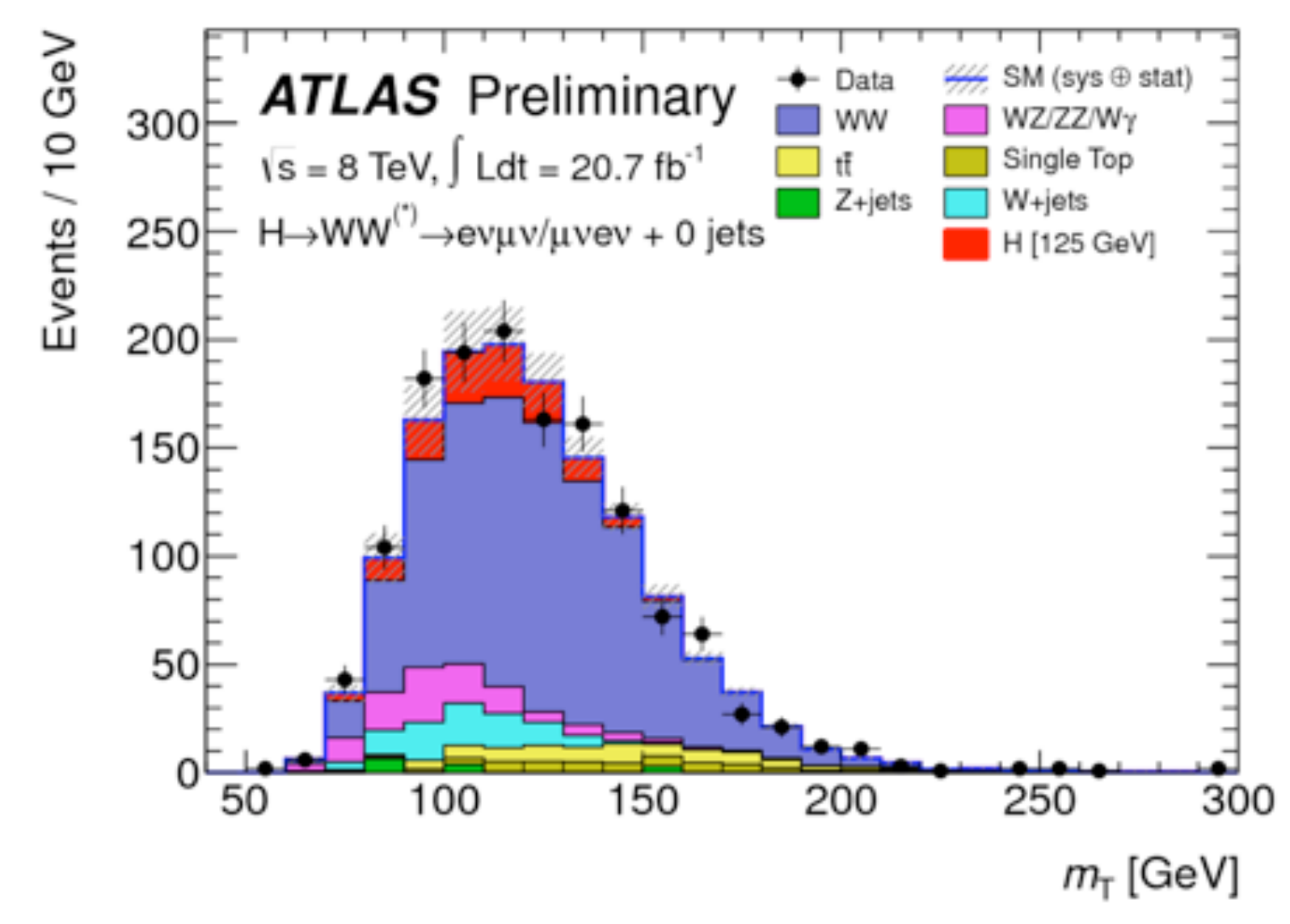

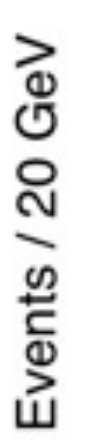

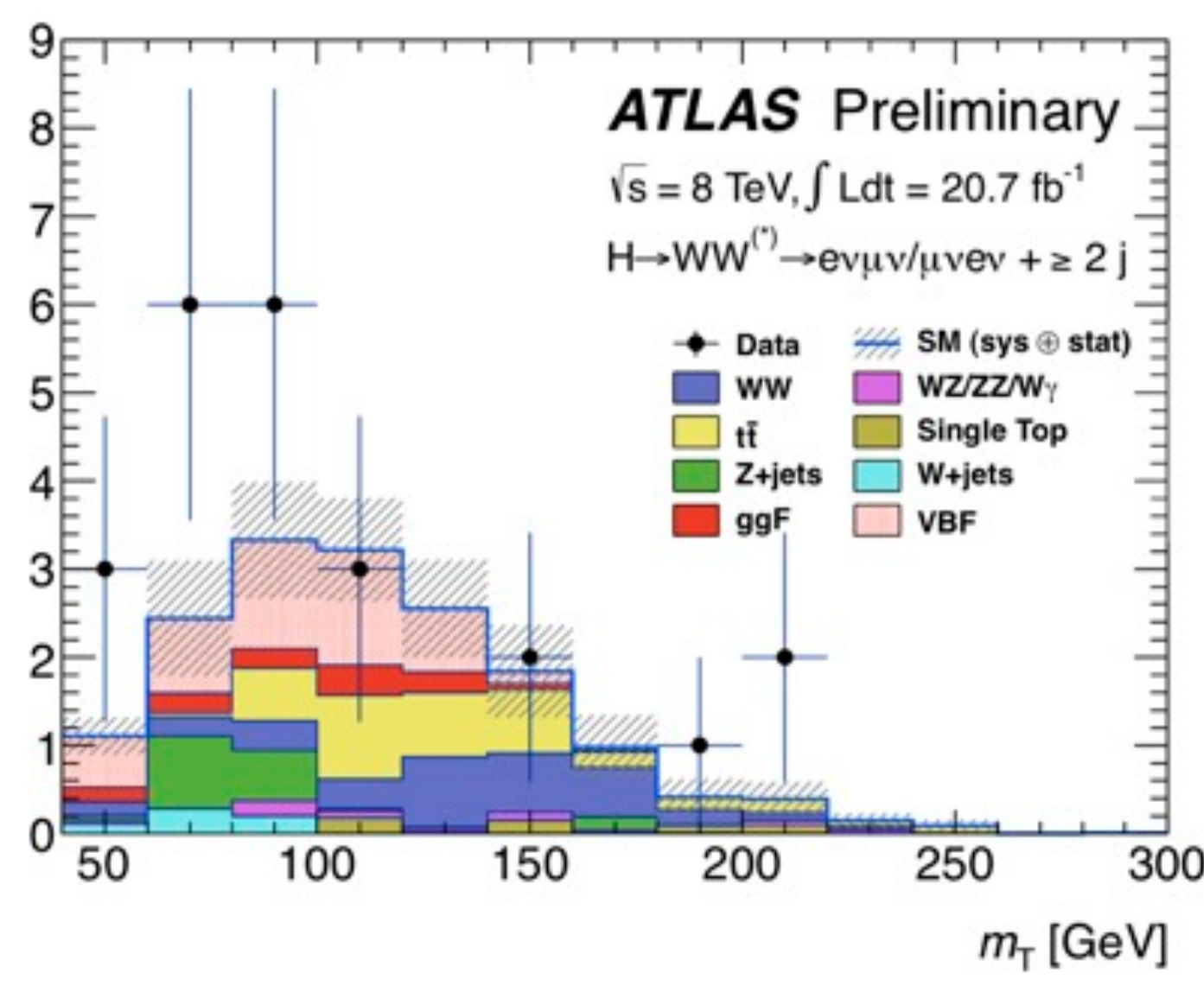




\section{Higgs Properties}

\section{HIGGS PROPERTIES}

- Mass Measurement

- Signal Strength and Couplings

- Spin 


\section{Signal Strength}

- Total signal strength $(\mu)$ tested in each channel (left)

- Signal strength grouped by common couplings: $g g F+t t H$ and $V B F+V H$ contributions
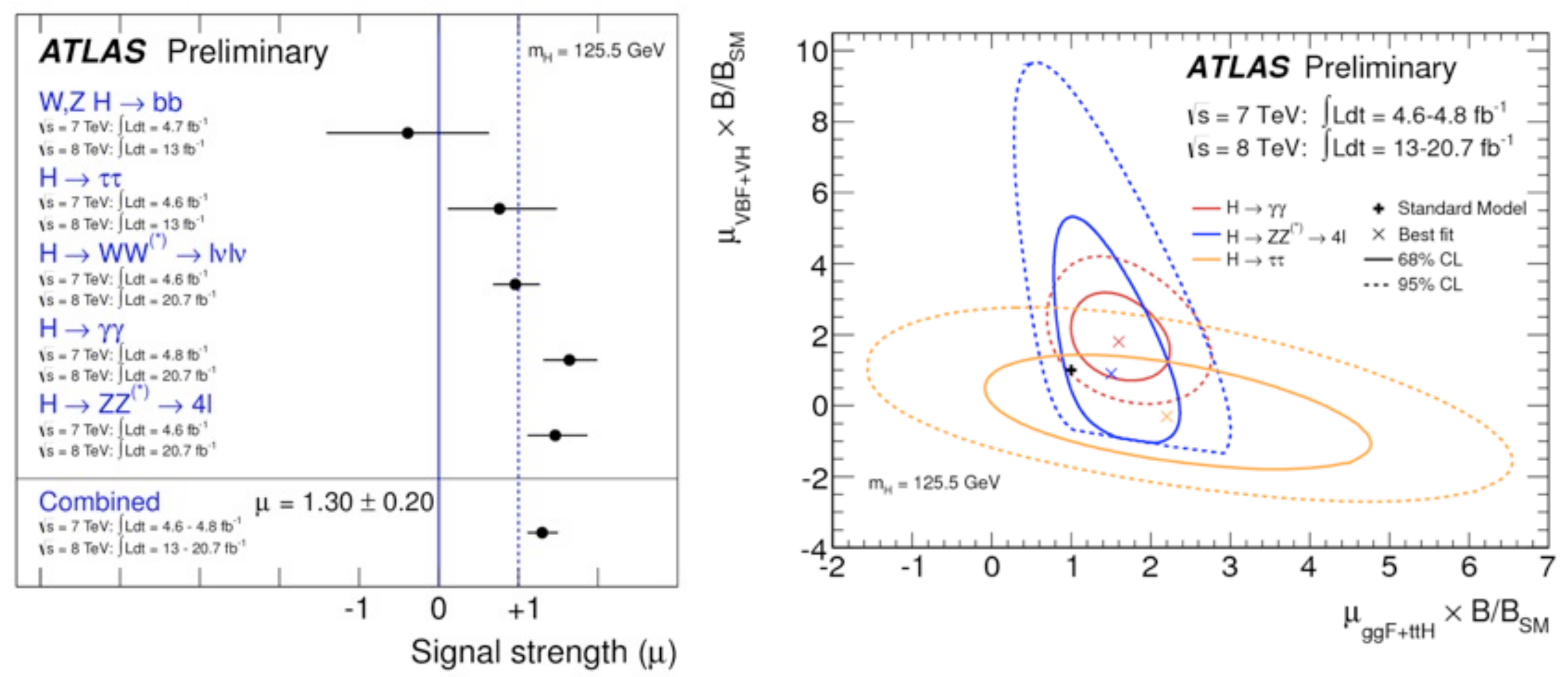

- Results are compatible with SM within $95 \% \mathrm{CL}$ bands

- $\mathrm{H} \rightarrow \mathrm{Yr}$ result a bit high 


\section{Couplings - Combination and Results}

- Define scale factors $K v$ for vector-boson and and $\mathrm{K}_{\mathrm{F}}$ for fermionic couplings

- $\sigma, B R$ proportional to $k^{2}$

- Left: couplings $K$ and coupling ratios $\lambda$
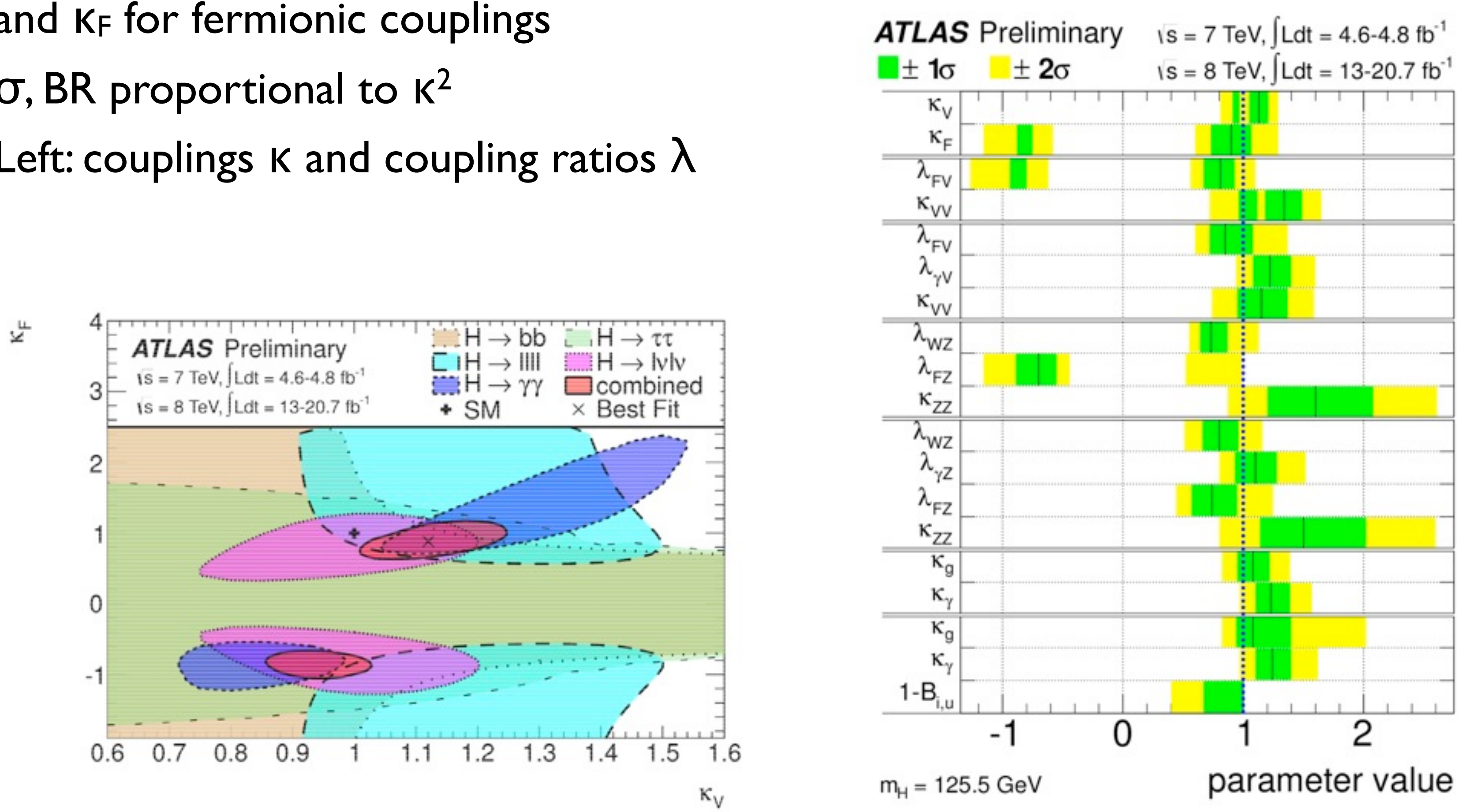


\section{General comments on Higgs spin analyses}

- The Higgs boson is a scalar particle - spin 0, positive parity

- Test new particle for spin-0, I, and 2 , as well as parity $(+,-)$

- Spin-I hypothesis is strongly disfavored due to particle's coupling to diphoton final state (Landau- Yang theorem)

- Define simplified graviton-like spin-2+ tensor, with only red boxes nonzero:

$$
\begin{aligned}
& A(H \rightarrow V V)=\Lambda^{-1} 22 g_{1} t_{\mu \nu} f^{* 1, \mu \alpha} f^{* 2, v \alpha}+2 g_{2} t_{\mu \nu} \frac{q_{\alpha} q_{\beta}}{\Lambda^{2}} f^{* 1, \mu \alpha} f^{* 2, \nu \beta} \\
& +g_{3} \frac{\tilde{q}^{\beta} \tilde{q}^{\alpha}}{\Lambda^{2}} t_{\beta \nu}\left(f^{* 1, \mu \nu} f_{\mu \alpha}^{* 2}+f^{* 2, \mu \nu} f_{\mu \alpha}^{* 1}\right)+g_{4} \frac{\tilde{q}^{\gamma} \tilde{q}^{\mu}}{\Lambda^{2}} t_{\mu v} f^{* 1, \alpha \beta} f_{\alpha \beta}^{*(2)} \\
& \left.\left.+m_{V}^{2}\left(2 g_{5} t_{\mu \nu} \epsilon_{1}^{* \mu} \epsilon_{2}^{* v}\right]+2 g_{6} \frac{\tilde{q}^{\mu} q_{\alpha}}{\Lambda^{2}} t_{\mu v} \epsilon_{1}^{* v} \epsilon_{2}^{* \alpha}-\epsilon_{1}^{* \alpha} \epsilon_{2}^{* \nu}\right)+g_{7} \frac{\tilde{q}^{\mu} \tilde{q}^{\nu}}{\Lambda^{2}} t_{\mu \nu} \epsilon_{1}^{*} \epsilon_{2}^{*}\right) \\
& +g_{8} \frac{\tilde{q}_{\mu} \tilde{q}_{v}}{\Lambda^{2}} t_{\mu v} f^{* 1, \alpha \beta} \tilde{f}_{\alpha \beta}^{*(2)}+g_{9} t_{\mu \alpha} \tilde{q}^{\alpha} \epsilon_{\mu \nu \rho \sigma} \epsilon_{1}^{* v} \epsilon_{2}^{* \rho} q^{\sigma} \\
& \left.+\frac{g_{10} t_{\mu} \tilde{q}^{\alpha}}{\Lambda^{2}} \epsilon_{\mu \nu \rho \sigma} q^{\rho} \tilde{q}^{\sigma}\left(\epsilon_{1}^{* \nu}\left(q \epsilon_{2}^{*}\right)+\epsilon_{2}^{* \nu}\left(q \epsilon_{1}^{*}\right)\right)\right] \text {, }
\end{aligned}
$$

- A 2+-spin particle can be produced in gluon-gluon fusion and $q \bar{q}$ annihilation processes

- Test various fractions of production processes $f_{g g}, f_{\mathrm{qq}}$

- i.e. $\mathrm{fg}_{\mathrm{gg}}$ increased in steps of $25 \%$ from $0 \%$ to $100 \%$

ATLAS-CONF-2013-040 


\section{Spin Hypothesis Measurement}

- $\mathrm{H} \rightarrow \mathrm{\gamma \gamma}$ Spin

- $|\cos \theta *|$, where $\theta^{*}$ is polar angle with respect to $z$-axis of the Collins-Soper (CS) frame

- Use mass as a discriminating variable as well

- Perform a maximum likelihood fit using two hypotheses

- $\mathrm{H} \rightarrow \mathrm{ZZ}$ Spin

- Discriminating variables are decay angles and intermediate $Z$ boson masses

- Train Boosted decision trees with both hypotheses

- Other dedicated spin analyses to test for 0+, 0-, 2+, 2- hypotheses

- $\mathrm{H} \rightarrow \mathrm{WW}^{*} \rightarrow$ IVlv Spin

- More stringent cuts to reduce continuum WW and $Z / \gamma^{*}$ backgrounds

- Boosted decision trees trained on observable kinematic quantities

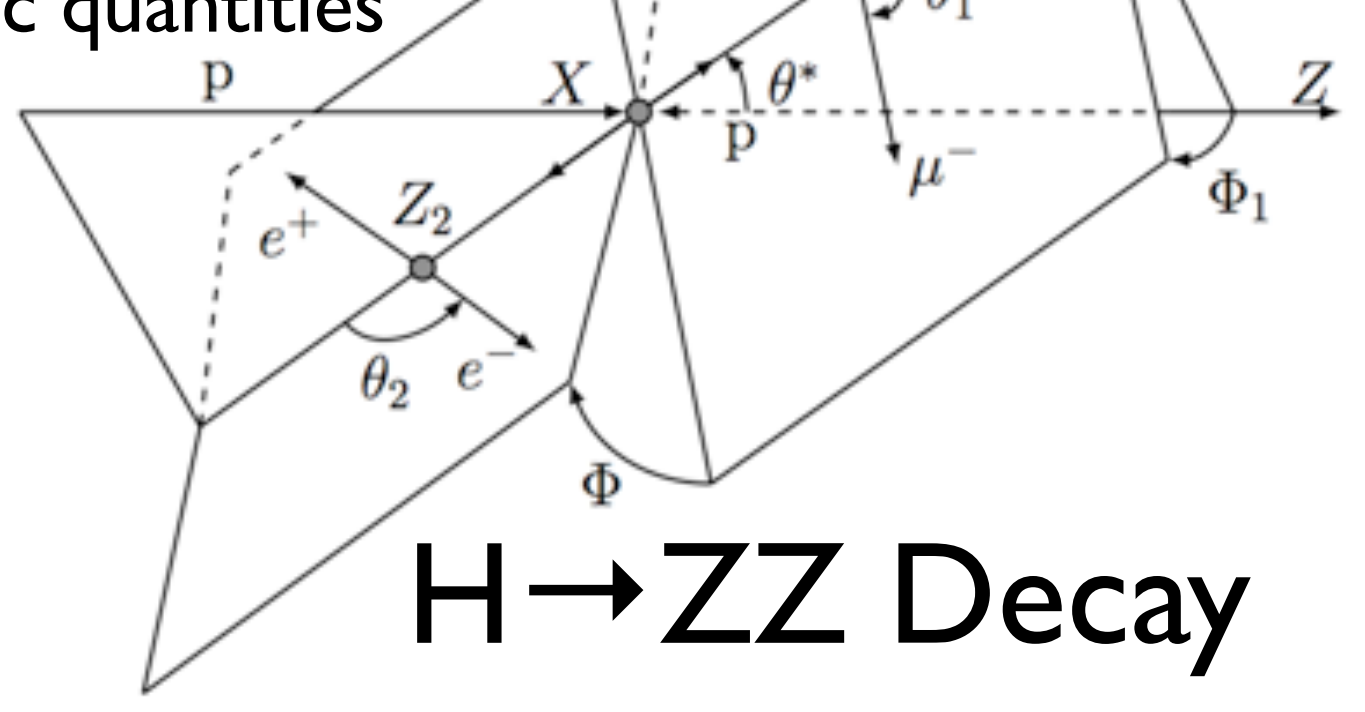




\section{Spin Combination - Results}
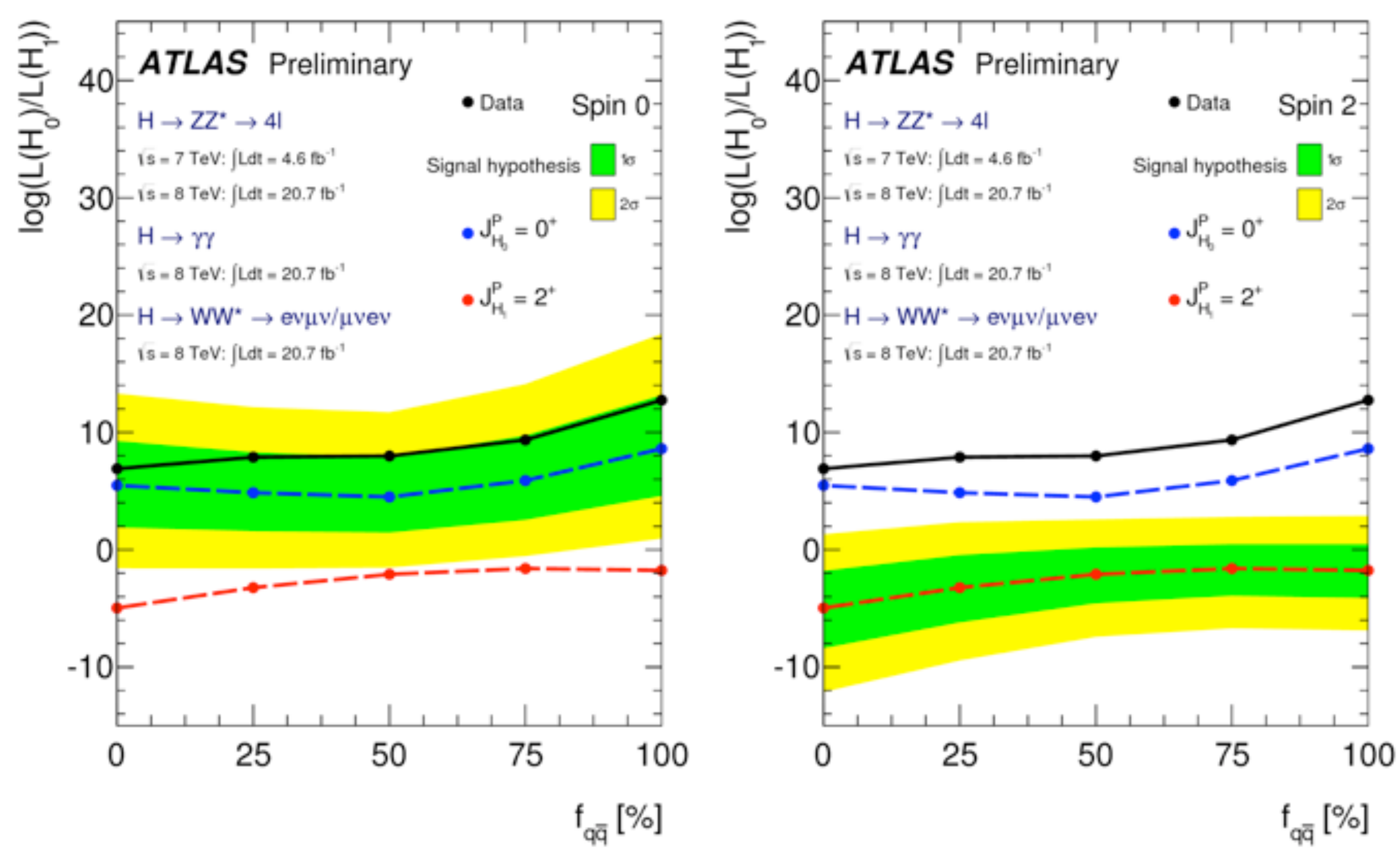

- Results reported in $25 \%$ steps of $\mathrm{f}_{\mathrm{qq}} \overline{\mathrm{q}}$

- Exclude this specific spin-2+ hypothesis with $99.9+\%$ confidence level

- Combined with information from individual channels: spin 0, even parity particle favored (consistent with SM Higgs Boson) 


\section{Additional Decay Modes}

- Yet to verify direct coupling to fermions

- Probe for possible new physics via invisible particles coupling to the Higgs

- Featured here:

- $\mathrm{H} \rightarrow \mathrm{TT}$

- $\mathrm{H} \rightarrow \mu \mu$

- $\mathrm{ZH} \rightarrow \ell \ell+$ Invisible

- $\mathrm{H} \rightarrow \mathrm{b} \bar{b}$

- Work on other decay modes; searches for high-mass Higgs, etc. 
- Taus decay leptonically ( $\mathrm{T}_{\text {lep }}$ ) or hadronically ( $T_{\text {had }}$ ) in the beampipe

- Weak tau decay leads to missing energy from neutrinos

- Reconstruct mass by combining visible kinematics with missing energy from neutrinos

- Missing Mass Calculator

- Analysis split into different final states, categorized to separate bkgs, production modes

- Results

- Combined limit: I.9x SM prediction

- Separate limits on VBF and non-VBF categories

- Search does not use full dataset: $4.7+13 \mathrm{fb}^{-1}$

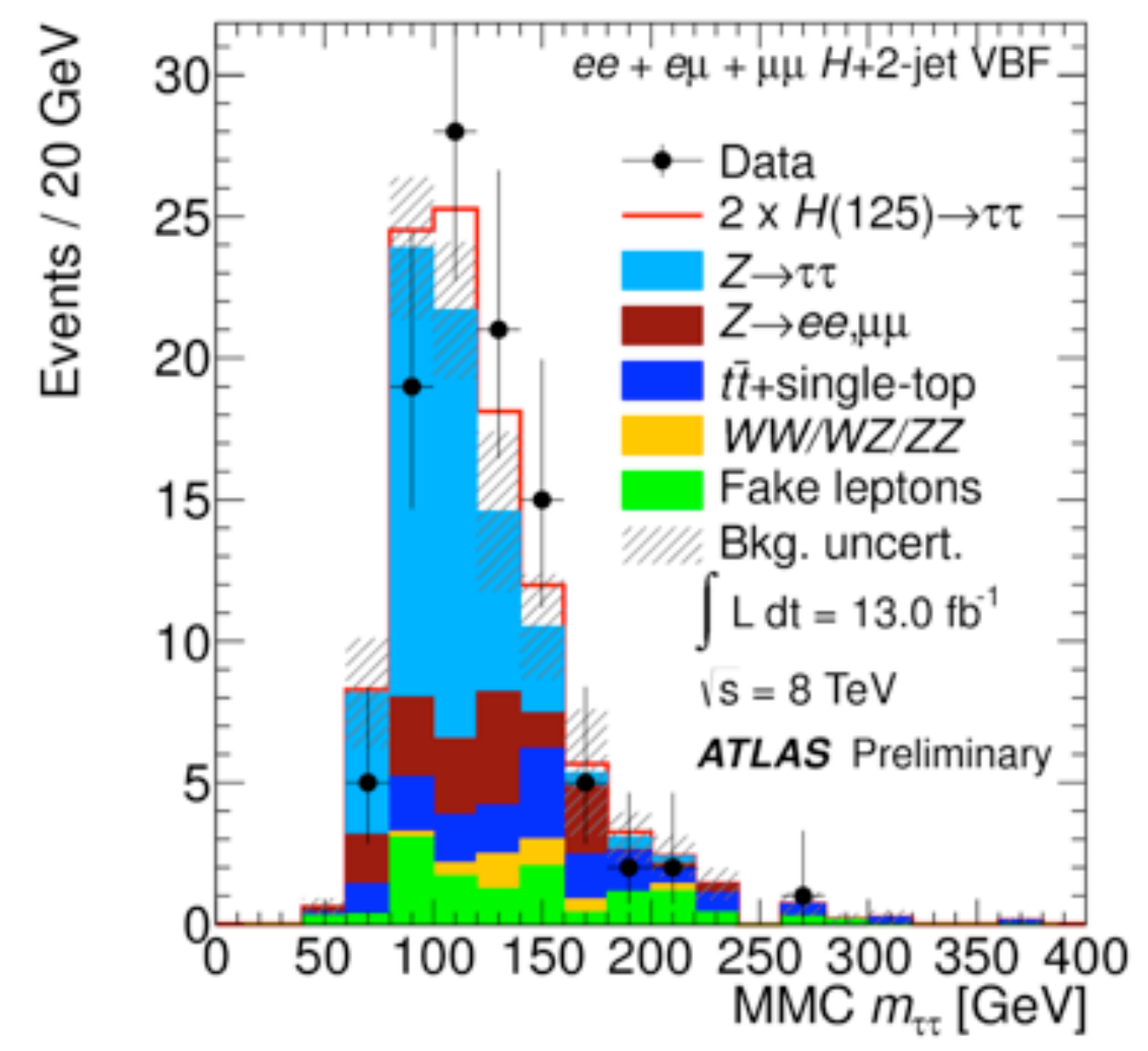




\section{$\mathrm{H} \rightarrow \mu \mu$ Channel}

Penn

- Only 2nd gen. fermion coupling measurement

- Dominant bkg: $Z / \gamma^{*} \rightarrow \mu \mu$ decays

- Search for possible enhancements to SM

- $\mathrm{H} \rightarrow \mu \mu \mathrm{BR}$ Measurements

- Current limit at 95\% CL: 9.8x SM prediction
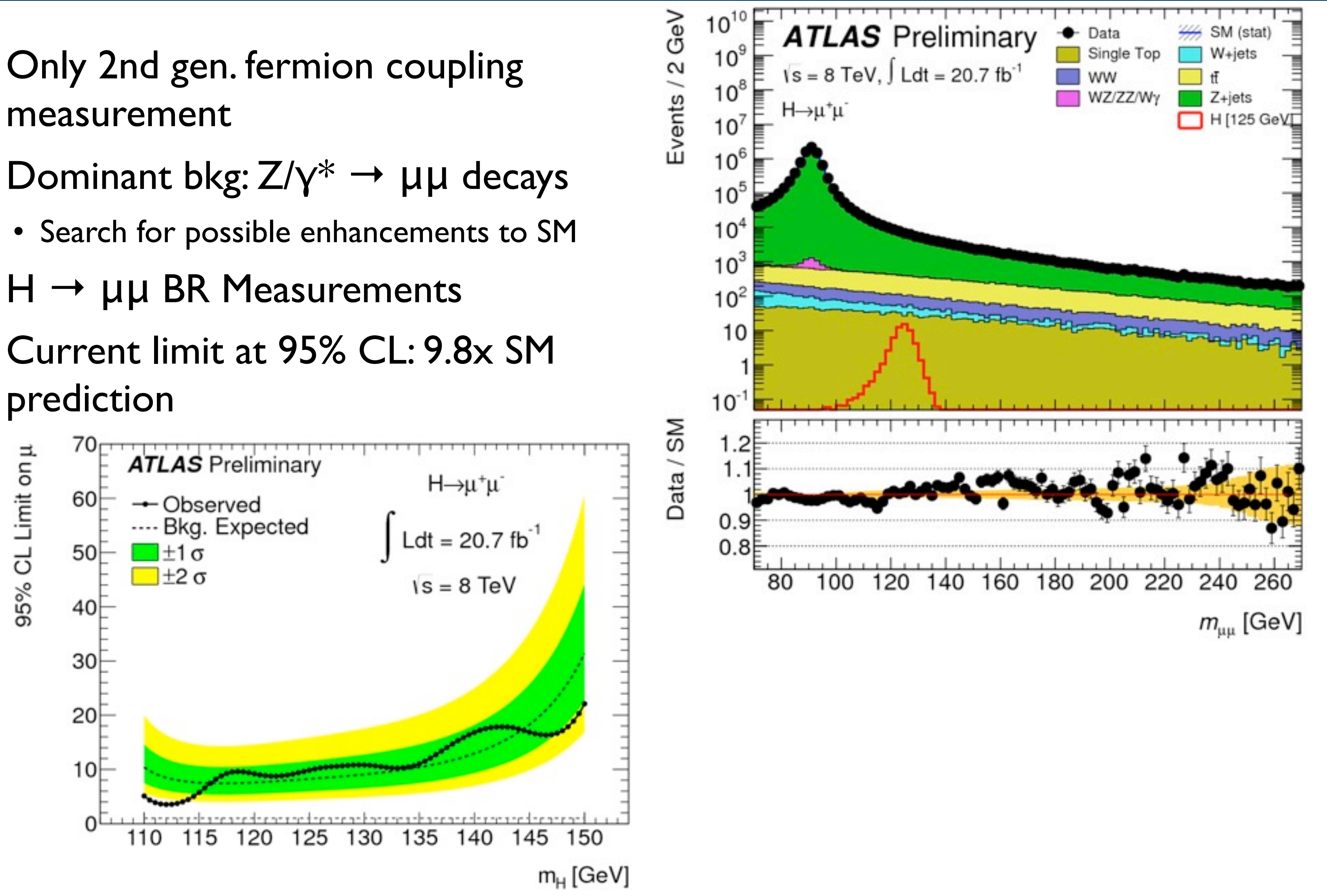
- $\mathrm{H} \rightarrow \mathrm{ZZ} \rightarrow 4 \mathrm{~V}$ BR is $4 \times 10^{-2}$ - too small

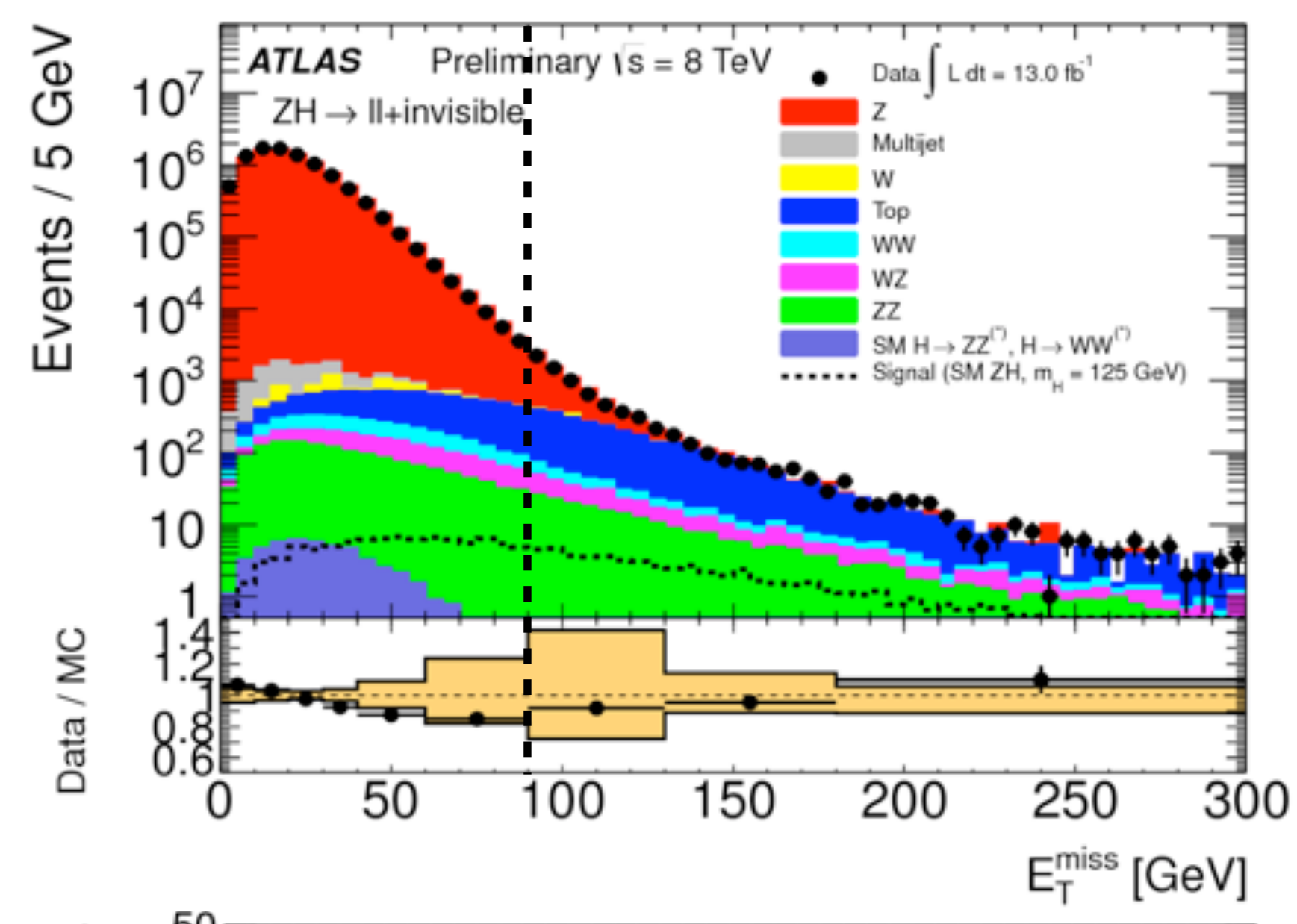

- Search for $Z$ decaying to $\mu \mu$ or ee

- Dilepton mass requirement to find $Z$

- Require >90 GeV Missing Transverse Momentum

- Additional kinematic cuts

- Place limit on total Higgs branching fraction to invisible at $65 \%$

- Search does not use full dataset: $4.7+13 \mathrm{fb}^{-1}$

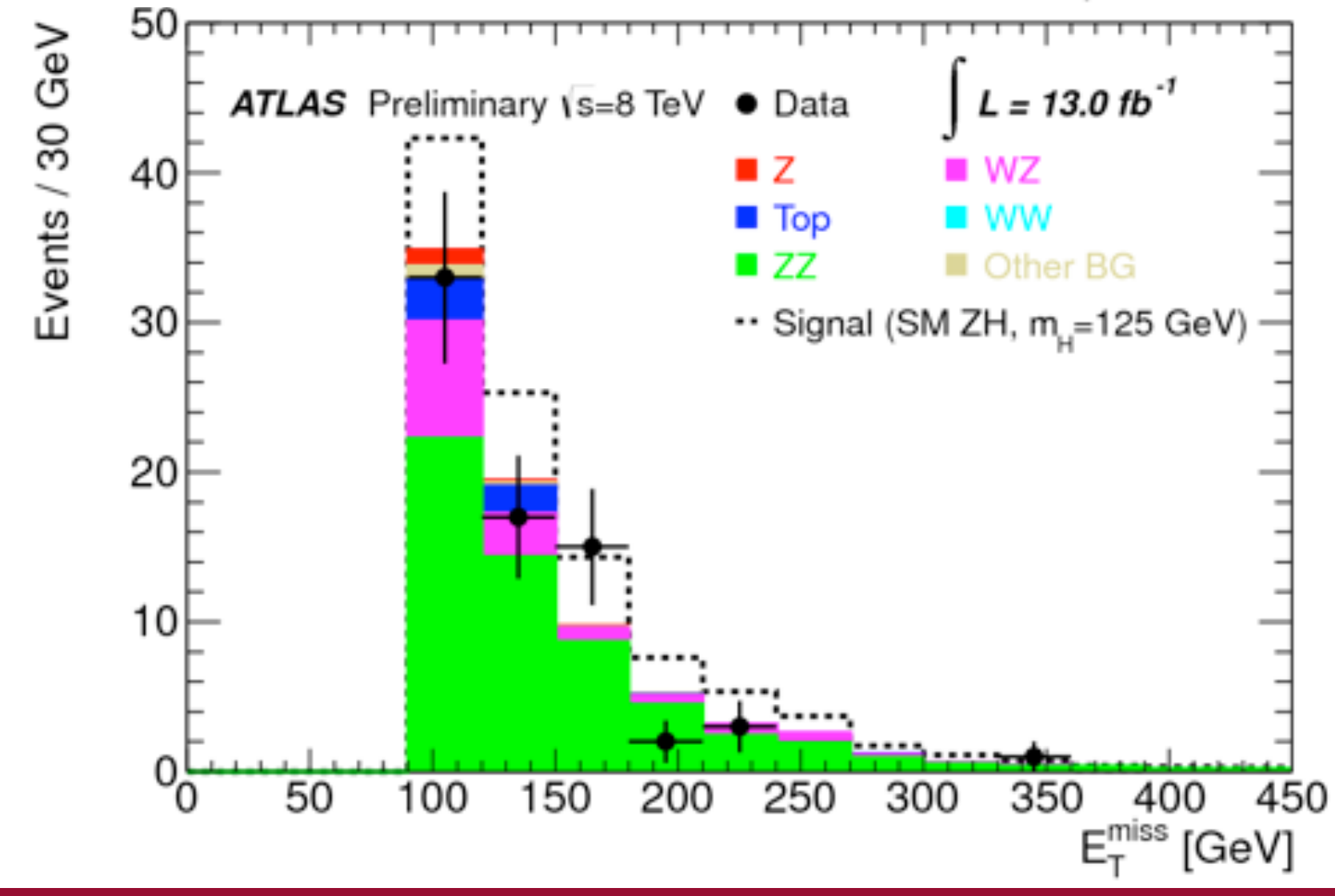




\section{$H \rightarrow b \bar{b}(V H, H \rightarrow b \bar{b})$}

- $\mathrm{H} \rightarrow \mathrm{b} \overline{\mathrm{b}}$ expected to have highest BR: $58 \%$

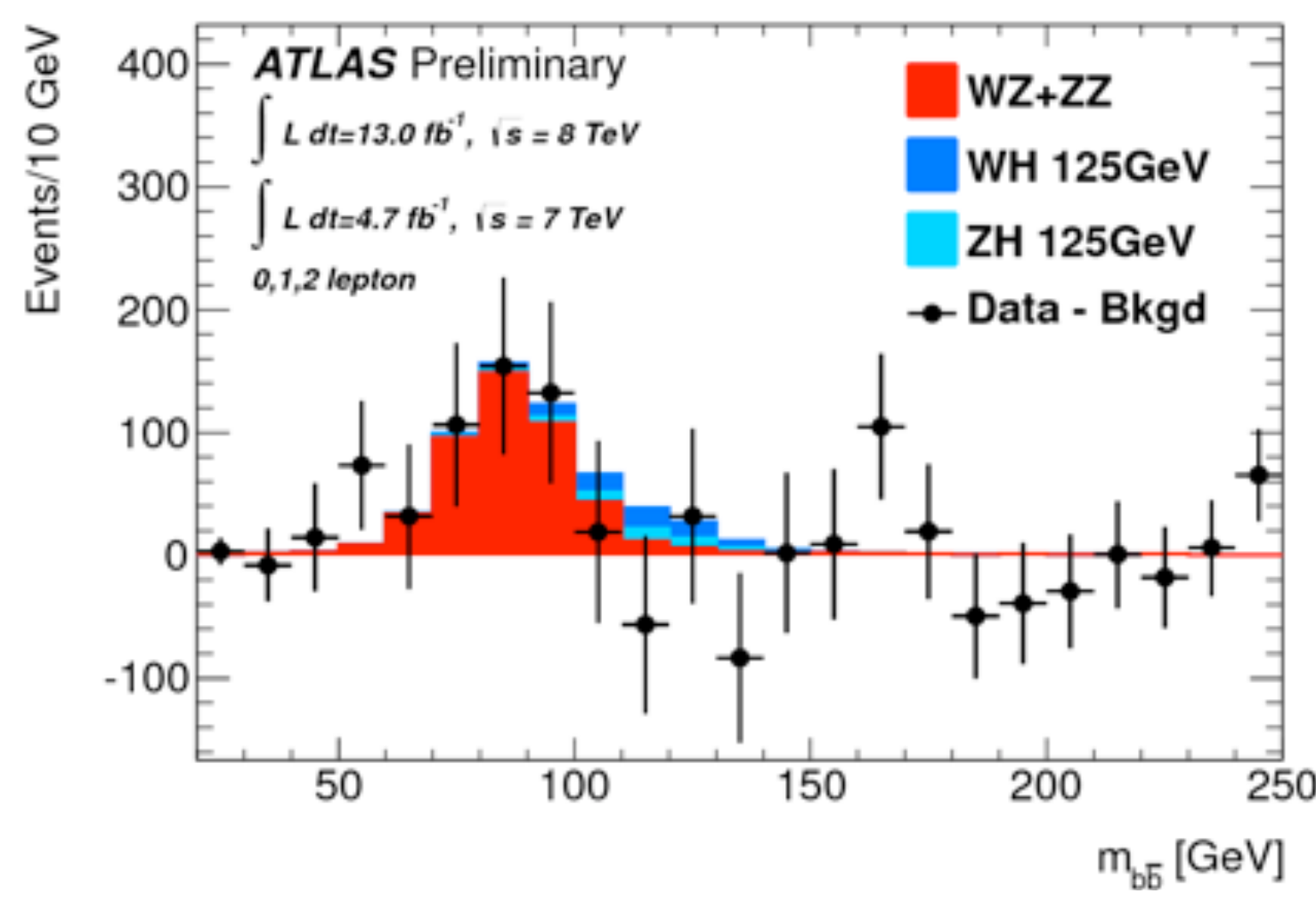

- Probed using associated production channels

- 3 decay modes:

- $\mathrm{ZH} \rightarrow \mathrm{vvb \overline {b }}$

- $\mathrm{ZH} \rightarrow \| \mathrm{lb} \bar{b}$

- $\mathrm{WH} \rightarrow \mid \mathrm{vb} \overline{\mathrm{b}}$

- Split into 0-, I-, 2-lepton categories

- Further categorized by $\mathrm{n}_{\text {jets }}$ and vector boson PT

- Approaching Ix SM exclusion limit

- Observed 95\% CL: I.8x SM prediction

- Search does not use full dataset: $4.7+13 \mathrm{fb}^{-1}$

ATLAS-CONF-2012-161

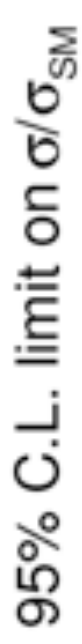




\section{Conclusions}

"Particle consistent with the Standard Model Higgs Boson"

- Further confirmation of Higgs boson

- Probing of properties (spin, couplings, signal strength)

- Measurement of VBF and ggF production modes, properties

- Closing in on fermionic final states

Exciting prospects for further measurements of the Higgs Boson

- End of 2012: LHC shut down

- 20I5: I4 TeV beam intensity, 5x luminosity

- Must contend with even harsher pileup conditions

- Run I: collected $\sim 25 \mathrm{fb}^{-1}$

- Run II: could collect up to $\sim 80 \mathrm{fb}^{-1}$ 


\section{BONUS}

\section{BONUS}




\section{Mass Measurement / discrepancy}

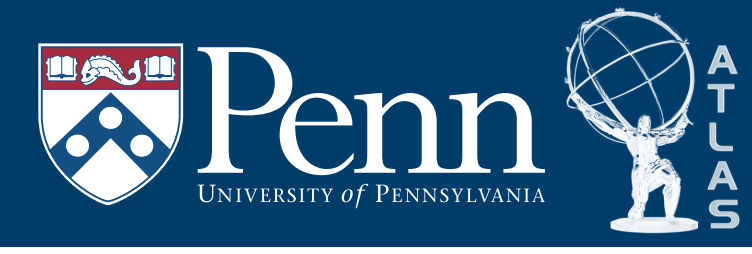

- $\Delta \mathrm{m}_{\mathrm{H}}=2.3$

- +0.6-0.7 stat

- +-0.6 sys

- Probability: I.2\% (2.5б)
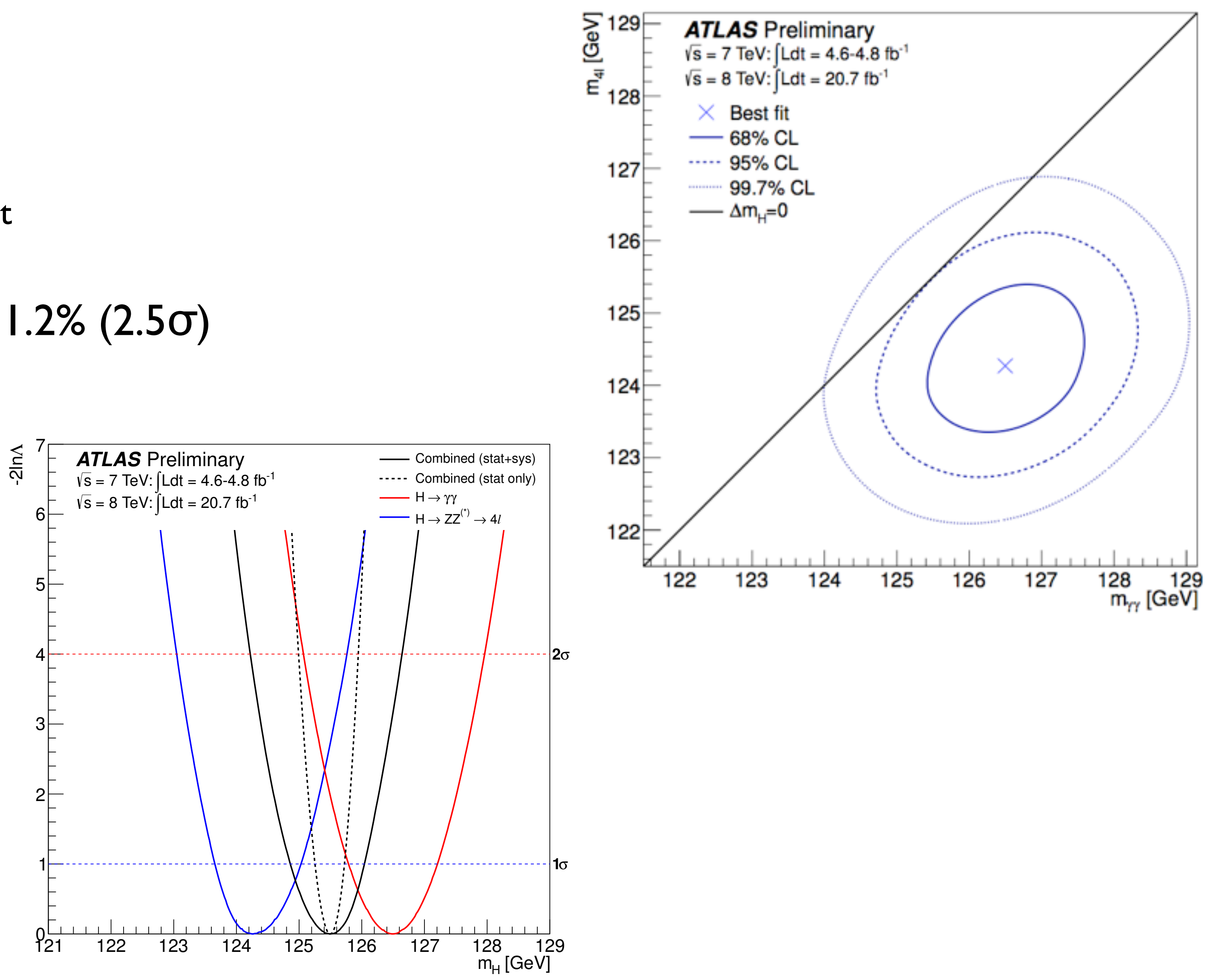
